\title{
A sharp upper bound for the size of Lusztig series
}

\author{
Christine Bessenrodt* and Alexandre Zalesski \\ Communicated by Britta Späth
}

\begin{abstract}
The paper is concerned with the character theory of finite groups of Lie type. The irreducible characters of a group $G$ of Lie type are partitioned in Lusztig series. We provide a simple formula for an upper bound of the maximal size of a Lusztig series for classical groups with connected center; this is expressed for each group $G$ in terms of its Lie rank and defining characteristic. When $G$ is specified as $G(q)$ and $q$ is large enough, we determine explicitly the maximum of the sizes of the Lusztig series of $G$.
\end{abstract}

\section{Introduction}

Let $\mathbf{G}$ be a reductive connected algebraic group. Let $F$ be a Frobenius (or Steinberg) endomorphism of $\mathbf{G}$ and $G:=\mathbf{G}^{F}=\{g \in G: F(g)=g\}$. Then $G$ is called a finite reductive group.

Let $\mathbf{G}^{*}$ denote the dual group of $\mathbf{G}$; see [3] or [5]. Then there is a Frobenius endomorphism $F^{*}$ of $\mathbf{G}^{*}$ which defines a finite group

$$
G^{*}=\mathbf{G}^{* F^{*}}=\left\{g \in G^{*}: F^{*}(g)=g\right\} \quad \text { with }|G|=\left|G^{*}\right| .
$$

The group $G^{*}$ is called the dual group of $G$ and plays an important role in the character theory of $G$. In particular, the Deligne-Lusztig theory partitions the set of irreducible characters of $G$ as a disjoint union of the so called Lusztig (geometric) series $\mathcal{E}_{S}$, where $s$ runs through a set of representatives in $G^{*}$ of the geometric conjugacy classes of semisimple elements of $\mathbf{G}^{*}$; see [5, Definition 13.16]. The characters from $\mathscr{E}_{1}$ (that is, for $s=1$ ) are called unipotent.

One of the questions not yet answered in the framework of the character theory of finite reductive groups is how large a Lusztig series can be. This has already attracted some attention in the literature; in particular, one needs to have a uniform upper bound for $\left|E_{S}\right|$. Liebeck and Shalev [10, Lemma 2.1] obtained the bound $\left|\varepsilon_{S}\right|<|W|^{2}$, where $W$ is the Weyl group of $\mathbf{G}$, and used this to bound the number of irreducible character degrees of $G$, as well as for proving some asymptotic results. This bound has been later improved to $\left|\mathcal{E}_{S}\right| \leq|W|$ in [14, Theorem 8.2].

In this paper, we obtain a sharp upper bound for $\max _{S}\left|\mathcal{E}_{S}\right|$ in terms of the rank of $\mathbf{G}$, where $\mathbf{G}$ is a simple algebraic group of classical type with trivial center (and 
$s$ ranges over the semisimple elements of $\left.G^{*}\right)$. In this case, $\left|\mathcal{E}_{s}\right|$ equals the number of unipotent characters of the group $C_{G^{*}}(s)$ (see [5, Theorem 13.23]). In fact, we compute the maximum of the number of unipotent characters of $C_{G^{*}}(s)$ when $\mathbf{G}^{*}$ is a simple simply connected algebraic group of classical type. More precisely, we compute the maximum of $\left|\mathcal{E}_{s}\right|$ for $G=G(q)$ with $q$ large enough (where $q$ is the well-known field parameter; usually, $q>n-9$ for $q$ even and $q>n-27$ for $q$ odd, where $n$ is the rank of $\mathbf{G})$.

To illustrate the nature of the problem, assume that $G=\operatorname{GL}_{n}(q)$. Then $G \cong G^{*}$. If $s=1$, then $C_{G^{*}}(s) \cong G$. The number of characters in $\mathscr{E}_{1}$ is well known to equal $p(n)$, the number of partitions of $n$. One could expect that $\left|\varepsilon_{s}\right| \leq p(n)$ for every $s$. However, such a conjecture is false, and the question of a sharp uniform upper bound for $\left|\mathcal{E}_{S}\right|$ does not have any obvious answer. One can refine this by asking for which $s$ the number of unipotent characters of $C_{G^{*}}(s)$ is maximal.

In this paper, we answer this question by determining the explicit value of the maximum for every classical group (for $q$ large) and describe $s$ for which the maximum is attained. Note that it is not a priori clear at all whether the above question is feasible and can have any precise answer. The content of this paper is in computing the maximum of certain combinatorial functions to which the original problem is reduced. It is interesting and somehow surprising that the formulae we obtain for $\max _{S}\left|\mathscr{E}_{S}\right|$ are much simpler than those available for the number of unipotent characters of $G$ and $G^{*}$ (see $[11, \S 3]$ ).

We expect that our results have a certain conceptual significance and will be useful for applications, in particular, for improving known upper bounds for the sum of the character degrees of $G$ (see [9, Chapter 5] and [14]).

Theorem 1.1. Let $\mathbf{G}$ be a simple algebraic group of rank $n$ of adjoint type in defining characteristic $p$ and $G=\mathbf{G}^{F}$, a finite reductive group. Then the size of a Lusztig series of $G$ does not exceed $c \cdot 5^{n / 4}$ for some constant $c$ bounded as follows (and specified explicitly in the detailed results below):

\begin{tabular}{llllll}
\hline$A_{n}$ & $C_{n}$ & $D_{n}$ & $B_{n}$ & $C_{n}$ & $D_{n}$ \\
& $p$ even & $p$ even & $p$ odd & $p$ odd & $p$ odd \\
$c<1.5$ & $c<15$ & $c<6$ & $c<95$ & $c<209$ & $c<44$ \\
\hline
\end{tabular}

We do not deal with the groups $\mathbf{G}$ of exceptional Lie type in defining characteristic $p$, and with $G={ }^{3} D_{4}(q)$, as in these cases, the sizes of Lusztig series are bounded by a constant which can be easily computed. For the other groups, the constant $c$ depends on the defining characteristic $p$ of $\mathbf{G}$, on the congruence of $n$ modulo 4 and, in case $D_{n}$, from the choice of the Frobenius endomorphism, which 
defines the groups $D_{n}^{+}(q)$ or $D_{n}^{-}(q)$. The fact that the above bound is sharp (with specified values of $c$ ) can be seen from Theorem 1.5 below, which provides an explicit value of the maximum size of a Lusztig series for $q$ large enough and for each type of the group $G$. In addition, this highlights the nature of the constant $c$ and reveals that the precise value of $c$ in each case depends on the residue of $n$ modulo 4 , a phenomenon which could not be expected in advance.

Our starting point is a result of Bessenrodt and Ono [1]. Let $\beta(n)$ be the maximal number of the form $p(\mu):=\prod_{j} p\left(\mu_{j}\right)$, where $\mu=\left(\mu_{1}, \ldots, \mu_{j}, \ldots\right)$ is a partition of $n$ and $p\left(\mu_{j}\right)$ is the number of partitions of $\mu_{j}$.

Theorem 1.2 ([1]). For $n=1, \ldots, 7$, we have $\beta(n)=p(n)=1,2,3,5,7,11,15$, respectively. Let $\pi(n)$ denote a partition $\mu$ of $n$ such that $\beta(n)=p(\mu)$. Then the partition $\pi(n)$ is uniquely determined for $n \neq 7$, whereas $\beta(7)$ is attained only at the two partitions $(7)$ and $(4,3)$.

For all $n \neq 1,2,3,7$, we have the following values for $\pi(n)$ and $\beta(n)$ :

\begin{tabular}{lll}
\hline$n \bmod 4$ & $\pi(n)$ & $\beta(n)$ \\
\hline 0 & $\left(4^{n / 4}\right)$ & $\beta(4)^{n / 4}=5^{n / 4}$ \\
1 & $\left(4^{(n-5) / 4}, 5\right)$ & $\beta(5) \beta(n-5)=7 \cdot 5^{(n-5) / 4}$ \\
2 & $\left(4^{(n-6) / 4}, 6\right)$ & $\beta(6) \beta(n-6)=11 \cdot 5^{(n-6) / 4}$ \\
3 & $\left(4^{(n-11) / 4}, 5,6\right)$ & $\beta(5) \beta(6) \beta(n-11)=77 \cdot 5^{(n-11) / 4}$ \\
\hline
\end{tabular}

Table 1

In particular, we always have $\beta(n) \leq 5^{n / 4}$.

Using Theorem 1.2, we obtain the following statements.

Theorem 1.3. If $G=\mathrm{GL}_{n}(q)$ or $\mathrm{U}_{n}(q)$, then the size of a Lusztig series does not exceed $\beta(n)$, and the bound $\beta(n)=5^{n / 4}$ is attained if $4 \mid n$ and $q>\frac{n}{4}$.

Our results for the other classical groups are more complex.

Let $\mathbf{G}$ be a simple classical algebraic group of adjoint type and $F$ a Frobenius endomorphism such that $G=\mathbf{G}^{F}$ is one of the groups $\operatorname{SO}_{2 n+1}(q), \operatorname{PCSp}_{2 n}(q)$, $\left(\mathrm{PSO}^{o}\right)_{2 n}^{ \pm}(q)$ in the notation of [12, Table 22.1]. Let $G^{*}$ be the dual group of $G$, so $G^{*}$ is $\operatorname{Sp}_{2 n}(q), \operatorname{Spin}_{2 n+1}(q)$ and $\operatorname{Spin}_{2 n}^{ \pm}(q)$, respectively. For small $n$, our results, stated in Proposition 1.4, are obtained by straightforward computer computations. For large $n$, a sharp upper bound for the number of unipotent characters of $C_{G^{*}}(s)$ and hence for the size of Lusztig series $\mathcal{E}_{s}$ of $G$ when $s$ ranges over the semisimple elements of $G^{*}$ is provided by Theorem 1.5 below. 
Let $\alpha(m), \alpha^{+}(m)$ and $\alpha^{-}(m)$ denote the number of unipotent characters of $\operatorname{Sp}_{2 m}(q), \operatorname{Spin}_{2 m}^{+}(q)$ and $\operatorname{Spin}_{2 m}^{-}(q)$, respectively.

Proposition 1.4. The following statements hold.

(1) Suppose that $q$ is even and $n<18$. Then $\max _{s}|\mathcal{E}|_{s}=\alpha(n), \alpha^{ \pm}(n)$ for $G$ of type $C_{n}(q), D_{n}^{ \pm}(q)$, respectively.

(2) Suppose that $q$ is odd and $n<32$ and $n \neq 2,4,6$ if $G=D_{n}^{-}(q)$. Then

$$
\begin{aligned}
\max _{s}\left|\mathcal{E}_{S}\right|= & \max _{a}\left\{\alpha^{+}(a) \alpha(n-a)\right\}, \max _{a}\{\alpha(a) \alpha(n-a)\}, \\
& \max _{a}\left\{\alpha^{+}(a) \alpha^{+}(n-a)\right\}, \max _{a}\left\{\alpha^{-}(a) \alpha^{+}(n-a)\right\}
\end{aligned}
$$

for $G$ of type $B_{n}(q), C_{n}(q), D_{n}^{+}(q), D_{n}^{-}(q)$, respectively, where a ranges between 0 and $n$. The explicit value of the maximum in each case is given by Tables 3 and 4 .

Theorem 1.5. Let $G^{*} \in\left\{\operatorname{Spin}_{2 n+1}(q) ; \operatorname{Sp}_{2 n}(q) ; \operatorname{Spin}_{2 n}^{ \pm}(q)\right\}$. For a semisimple element $s \in G^{*}$, let $\mathcal{E}_{s}$ denote the Lusztig series of irreducible characters of $G$.

(1) Let $G^{*}=\operatorname{Sp}_{2 n}(q)$, $q$ even, or $\operatorname{Spin}_{2 n+1}(q)$, q even. For $n \geq 18$, we have $\left|\varepsilon_{s}\right| \leq f(n)$, where

$$
f(n)= \begin{cases}\alpha(16) \beta(n-16)=8988 \cdot 5^{(n-16) / 4} & \text { if } n \equiv 0 \bmod 4, \\ \alpha(15) \beta(n-15)=66396 \cdot 5^{(n-21) / 4} & \text { if } n \equiv 1 \bmod 4 \\ \alpha(14) \beta(n-14)=4020 \cdot 5^{(n-14) / 4} & \text { if } n \equiv 2 \bmod 4 \\ \alpha(15) \beta(n-15)=6036 \cdot 5^{(n-15) / 4} & \text { if } n \equiv 3 \bmod 4 .\end{cases}
$$

(2) Let $G^{*}=\operatorname{Spin}_{2 n}^{ \pm}(q)$, q even. For $n \geq 18$, we have $\left|\mathcal{E}_{s}\right| \leq f^{ \pm}(n)$, where

$$
\begin{aligned}
& f^{+}(n)= \begin{cases}\alpha^{+}(16) \beta(n-16)=4110 \cdot 5^{(n-16) / 4} & \text { if } n \equiv 0 \bmod 4, \\
\alpha^{+}(17) \beta(n-17)=6007 \cdot 5^{(n-17) / 4} & \text { if } n \equiv 1 \bmod 4, \\
\alpha^{+}(14) \beta(n-14)=1836 \cdot 5^{(n-14) / 4} & \text { if } n \equiv 2 \bmod 4, \\
\alpha^{+}(15) \beta(n-15)=2730 \cdot 5^{(n-15) / 4} & \text { if } n \equiv 3 \bmod 4,\end{cases} \\
& f^{-}(n)= \begin{cases}\alpha^{-}(16) \beta(n-16)=4066 \cdot 5^{(n-16) / 4} & \text { if } n \equiv 0 \bmod 4, \\
\alpha^{-}(17) \beta(n-17)=6007 \cdot 5^{(n-17) / 4} & \text { if } n \equiv 1 \bmod 4, \\
\alpha^{-}(14) \beta(n-14)=1806 \cdot 5^{(n-14) / 4} & \text { if } n \equiv 2 \bmod 4, \\
\alpha^{-}(15) \beta(n-15)=2730 \cdot 5^{(n-15) / 4} & \text { if } n \equiv 3 \bmod 4 .\end{cases}
\end{aligned}
$$


(3) Let $G^{*}=\operatorname{Sp}_{2 n}(q)$, q odd. For $n \geq 32$, we have $\left|\varepsilon_{S}\right| \leq \tau(n)$, where

$$
\tau(n)= \begin{cases}\alpha(14) \alpha(14) \beta(n-28)=16160400 \cdot 5^{(n-28) / 4} & \text { if } n \equiv 0 \bmod 4, \\ \alpha(15) \alpha(14) \beta(n-29)=24264720 \cdot 5^{(n-29) / 4} & \text { if } n \equiv 1 \bmod 4, \\ \alpha(15) \alpha(15) \beta(n-30)=36433296 \cdot 5^{(n-30) / 4} & \text { if } n \equiv 2 \bmod 4, \\ \alpha(16) \alpha(15) \beta(n-31)=54251568 \cdot 5^{(n-31) / 4} & \text { if } n \equiv 3 \bmod 4 .\end{cases}
$$

(4) Let $s \in G^{*}=\operatorname{Spin}_{2 n+1}(q)$, q odd. For $n \geq 32$, we have $\left|\mathcal{E}_{s}\right| \leq \theta(n)$, where

$$
\theta(n)= \begin{cases}\alpha(16) \alpha^{+}(16) \beta(n-32)=36940680 \cdot 5^{(n-32) / 4} & \text { if } n \equiv 0 \bmod 4, \\ \alpha(15) \alpha^{+}(14) \beta(n-29)=11082096 \cdot 5^{(n-29) / 4} & \text { if } n \equiv 1 \bmod 4, \\ \alpha(14) \alpha^{+}(16) \beta(n-30)=16522200 \cdot 5^{(n-30) / 4} & \text { if } n \equiv 2 \bmod 4, \\ \alpha(15) \alpha^{+}(16) \beta(n-31)=24807960 \cdot 5^{(n-31) / 4} & \text { if } n \equiv 3 \bmod 4 .\end{cases}
$$

(5) Let $G^{*}=\operatorname{Spin}_{2 n}^{ \pm}(q)$, q odd. For $n \geq 32$, we have $\left|\varepsilon_{s}\right| \leq \theta^{ \pm}(n)$, where

$\theta^{+}(n)= \begin{cases}\alpha^{+}(16) \alpha^{+}(16) \beta(n-32)=16892100 \cdot 5^{(n-32) / 4} & \text { if } n \equiv 0 \bmod 4, \\ \alpha^{+}(15) \alpha^{+}(14) \beta(n-29)=5012280 \cdot 5^{(n-29) / 4} & \text { if } n \equiv 1 \bmod 4, \\ \alpha^{+}(16) \alpha^{+}(14) \beta(n-30)=7545960 \cdot 5^{(n-30) / 4} & \text { if } n \equiv 2 \bmod 4, \\ \alpha^{+}(16) \alpha^{+}(15) \beta(n-31)=11220300 \cdot 5^{(n-31) / 4} & \text { if } n \equiv 3 \bmod 4,\end{cases}$

$\theta^{-}(n)= \begin{cases}\alpha^{-}(16) \alpha^{+}(16) \beta(n-32)=16711260 \cdot 5^{(n-32) / 4} & \text { if } n \equiv 0 \bmod 4, \\ \alpha^{-}(15) \alpha^{+}(14) \beta(n-29)=5012280 \cdot 5^{(n-29) / 4} & \text { if } n \equiv 1 \bmod 4, \\ \alpha^{-}(16) \alpha^{+}(14) \beta(n-30)=7465176 \cdot 5^{(n-30) / 4} & \text { if } n \equiv 2 \bmod 4, \\ \alpha^{-}(15) \alpha^{+}(16) \beta(n-31)=11220300 \cdot 5^{(n-31) / 4} & \text { if } n \equiv 3 \bmod 4 .\end{cases}$

In addition, the detailed bounds given in (1)-(5) are attained if $q>n-9$ if $q$ is even, and $q>n-27$ if $q$ is odd.

Note that Lusztig series were originally defined only for groups with connected center, but later, this notion has been extended to arbitrary connected reductive groups so that, again, the size of a Lusztig series equals the number of unipotent characters of $C_{G^{*}}(s)$; see [5, Theorem 13.23]. Then the number of unipotent characters of $C_{G^{*}}(s)$ does not exceed $\left|C_{G^{*}}(s): C_{G^{*}}(s)^{0}\right| \cdot v\left(C_{G^{*}}(s)^{0}\right)$, where

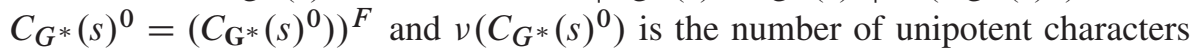
of $C_{G^{*}}(s)^{0}$. The index $\left|C_{G^{*}}(s): C_{G^{*}}(s)^{0}\right|$ does not exceed $r+1$ for groups of type $A_{r}$ and 4 for the other simple groups [15, Chapter II, Corollary 4.4]. So we can replace $c$ by $(r+1) c$ for the $A_{r}$-case and $4 c$ for the other groups (in fact, the latter is needed only for $q$ odd). However, these bounds may not be sharp. 
Our strategy can be outlined as follows. The simplest case is where $G=\mathrm{GL}_{n}(q)$ or $\mathrm{U}_{n}(q)$; here we show (Section 3 ) that $\left|\mathcal{E}_{s}\right| \leq \beta(n)$, and the bound is attained for $q$ large enough. For the other classical groups, this bound is valid only if \pm 1 are not eigenvalues of $s$ on the natural $\mathbb{F}_{q} G^{*}$-module $V$ for $G^{*}$ (Lemma 4.6). Suppose first that $G^{*}=\operatorname{Sp}_{2 n}(q), q$ odd, and $k, l$ are the multiplicities of the eigenvalues 1 and -1 , respectively, of $s$ on $V$. Then we show that

$$
\left|\mathcal{E}_{S}\right| \leq \max \alpha\left(\frac{k}{2}\right) \alpha\left(\frac{l}{2}\right) \beta(m), \quad \text { where } \frac{k+l}{2}+m=n .
$$

This reduces the problem to computing the above maximum, and next we show that the bound is attained for some $s$ if $q$ is large enough. If $G^{*}$ is an orthogonal group, then we have a similar reduction with $\alpha\left(\frac{k}{2}\right) \alpha\left(\frac{l}{2}\right)$ to be replaced by $\alpha^{ \pm}\left(\frac{k}{2}\right) \alpha^{ \pm}\left(\frac{l}{2}\right)$ if $\operatorname{dim} V$ is even, and $\alpha\left(\frac{k}{2}\right) \alpha^{ \pm}\left(\frac{l}{2}\right)$ if $\operatorname{dim} V$ is odd, with a certain choice of the signs. If $q$ is even, then we argue similarly. The maximum of the products in question is computed in Section 5. The proof of Theorem 1.5 occupies Sections 6 and 7 for $q$ even and odd, respectively.

Notation. The size of a finite set $S$ is denoted by $|S|$. Also, we write $|g|$ for the order of a group element, which does not lead to any confusion. For a group $G$, we denote by $Z(G)$ the center of $G$, and by $C_{G}(S)$ the centralizer of a subset $S$ of $G$ in $G$. We use this notation also in the situation where $V$ is a set on which $G$ acts by permutations or a vector space on which $G$ acts by linear transformations. So $C_{V}(S)=\{v \in V: s v=v$ for all $s \in S\}$. For $S \subset G$, we denote by $\langle S\rangle$ the subgroup generated by $S$.

$\operatorname{Id}_{n}$ is the identity $(n \times n)$-matrix. By $\operatorname{diag}\left(x_{1}, \ldots, x_{n}\right)$, we denote the diagonal matrix with subsequent diagonal entries $x_{1}, \ldots, x_{n}$. A similar notation is used for a block-diagonal matrix.

We denote by $\mathbb{N}$ the set of natural numbers. For $n \in \mathbb{N}, p(n)$ denotes the number of partitions of $n$; for a partition $\mu=\left(\mu_{1}, \ldots, \mu_{t}\right)$, we set $p(\mu)=\prod_{j=1}^{t} p\left(\mu_{j}\right)$. We then set $\beta(n)=\max p(\mu)$, where the maximum is taken over all partitions $\mu$ of $n$.

By $\mathbb{F}_{q}$, we denote the field of $q$ elements. If $K$ is a field, then $K^{\times}$denotes the multiplicative group of $K$ and $\bar{K}$ an algebraic closure of $K$.

All vector spaces considered in the paper are of finite dimension. By GL $(V)$, we denote the group of all invertible linear transformations of a vector space $V$. If the ground field $K$ is not algebraically closed and $s \in \mathrm{GL}(V)$ is a semisimple element, then the natural analog of eigenspaces are homogeneous components of $s$ on $V$; these are the sum of all minimal non-zero $K\langle s\rangle$-submodules of $V$ isomorphic to each other. If $s$ has a single homogeneous component on $V$, we say that $s$ is homogeneous. 
For an algebraic group $\mathbf{G}$, we denote by $\mathbf{G}^{0}$ the connected component of the identity of $\mathbf{G}$. We use $F$ to denote a Frobenius endomorphism of an algebraic group, and we often use it for different algebraic groups. We usually write $G$ for $\mathbf{G}^{F}=\{g \in \mathbf{G}: F(g)=g\}$. If $\mathbf{G}$ is connected reductive, we call $G=\mathbf{G}^{F}$ a finite reductive group. For a finite reductive group $G$, we denote by $\nu(G)$ the number of unipotent characters of $G$. See Section 2.2 for more details. As mentioned in the introduction, $\alpha(m), \alpha^{+}(m), \alpha^{-}(m)$ stands for the number of unipotent characters of $\operatorname{Sp}_{2 m}(q), \operatorname{Spin}_{2 m}^{+}(q), \operatorname{Spin}_{2 m}^{-}(q)$, respectively. By $\mathbf{G}^{*}$ and $G^{*}$, we denote the dual groups of a reductive algebraic group $\mathbf{G}$ and of a finite reductive group $G$, respectively.

Our notation for classical groups is standard, except for the special orthogonal groups of even characteristic; following [12], we denote by $\operatorname{SO}_{2 n}^{ \pm}(q)$ and $\mathrm{SO}_{2 n}\left(\overline{\mathbb{F}}_{q}\right)$ with $q$ even the subgroup of index 2 in the full orthogonal group $\mathrm{O}_{2 n}^{ \pm}(q)$ and $\mathrm{O}_{2 n}\left(\overline{\mathbb{F}}_{q}\right)$, respectively. (The advantage is that certain results can be stated uniformly for $q$ odd and even.) In addition, dealing with the groups $\mathrm{SO}_{2 n+1}(q)$, we assume that $q$ is odd as $\mathrm{SO}_{2 n+1}(q) \cong \operatorname{Sp}_{2 n}(q)$ whenever $q$ is even.

We expect a reader to be familiar with the geometry of classical groups; most necessary facts can be found in [8, Chapter 2]. In particular, for the notion of Witt defect of an orthogonal space, see [8, p. 28]. Nonetheless, we recall a few notions from this area.

An orthogonal space means a vector space $V$ of finite dimension over a field $K$, say, endowed with a non-degenerate symmetric bilinear form $f\left(v_{1}, v_{2}\right) \in K$ for $v_{1}, v_{2} \in V$, and if the characteristic of $K$ equals 2 , then the form is additionally assumed to be alternating (that is, $f(v, v)=0$ for $v \in V$ ) and non-defective [4, Chapter I, § 16]. The full orthogonal group is denoted by $\mathrm{O}(V)$. The spinor group of an orthogonal space $V$ (we call it the full spinor group of $V$ ) is defined in terms of the Clifford algebra of $V$ [4, Chapter II, §7]; this yields the notion of spinor norm, which defines the subgroup $\Omega(V)$ of $\mathrm{O}(V)$ formed by elements of spinor norm 1. In particular, if the ground field is of characteristic 2, we have $\Omega(V)=\operatorname{SO}(V)$ by convention. We use $\operatorname{Spin}(V)$ to denote the preimage of $\Omega(V)$ in the spinor group of $V$ under the natural projection of it onto $\mathrm{O}(V)$; see [4, Chapter II, §7].

\section{Preliminaries}

For later considerations, we will need the explicit formulae for $\beta(n)$ from [1] which we now recall.

\subsection{Some properties of the function $\beta(n)$}

From Theorem 1.2, we deduce a number of properties of the numbers $\beta(n)$. 
Lemma 2.1. Let $0<k \leq n$ be integers. Then

$$
\beta(k)<\beta(n) \quad \text { for } k<n \quad \text { and } \quad \beta(k) \beta(n) \leq \beta(k+n) .
$$

More precisely, if $k \neq 1,2,3,7$, then

$$
\frac{\beta(k+n)}{\beta(k) \beta(n)}= \begin{cases}1 & \text { if } k \equiv 0 \text { or } n \equiv 0 \bmod 4, \\ \frac{55}{49} & \text { if } k \equiv 1 \text { and } n \equiv 1 \bmod 4, \\ 1 & \text { if } k \equiv 1 \text { and } n \equiv 2 \operatorname{or} k \equiv 2 \text { and } n \equiv 1 \bmod 4, \\ \frac{625}{539} & \text { if } k \equiv 1 \text { and } n \equiv 3 \operatorname{or} k \equiv 3 \text { and } n \equiv 1 \bmod 4, \\ \frac{125}{121} & \text { if } k \equiv 2 \text { and } n \equiv 2 \bmod 4, \\ \frac{125}{77} & \text { if } k \equiv 2 \text { and } n \equiv 3 \operatorname{or} k \equiv 3 \text { and } n \equiv 2 \bmod 4, \\ \frac{625}{539} & \text { if } k \equiv 3 \text { and } n \equiv 3 \bmod 4 .\end{cases}
$$

For $k=1,2,3,7$ and $n>7$, the values of $\frac{\beta(n+k)}{\beta(k) \beta(n)}$ are as follows:

\begin{tabular}{lcccc}
\hline$n$ & $k=1$ & $k=2$ & $k=3$ & $k=7$ \\
\hline$n \equiv 0 \bmod 4$ & $\frac{7}{5}$ & $\frac{11}{10}$ & $\frac{77}{75}$ & $\frac{77}{75}$ \\
$n \equiv 1 \bmod 4$ & $\frac{11}{5}$ & $\frac{11}{10}$ & $\frac{25}{21}$ & $\frac{25}{21}$ \\
$n \equiv 2 \bmod 4$ & $\frac{7}{5}$ & $\frac{25}{22}$ & $\frac{35}{33}$ & $\frac{35}{33}$ \\
$n \equiv 3 \bmod 4$ & $\frac{125}{77}$ & $\frac{25}{22}$ & $\frac{25}{21}$ & $\frac{25}{21}$ \\
\hline
\end{tabular}

Proof. Straightforward computations using Theorem 1.2.

Lemma 2.2. Let $n>2$ be an integer.

(1) If $n$ is even, then $\beta\left(\frac{n}{2}\right)<\beta(3) \beta(n-3)=3 \beta(n-3)$, and for $n>4$, we have $\beta\left(\frac{n}{2}\right)<\beta(5) \beta(n-5)=7 \beta(n-5)$.

(2) We have $5^{(n-3) / 4}<\beta(n)$.

Proof. (1) If $\frac{n}{2} \leq n-5$, then $\beta\left(\frac{n}{2}\right) \leq \beta(n-5)<\beta(n-3)$ by Lemma 2.1. Otherwise, $n<10$, and the claim follows by inspection (Table 2 ).

(2) We have $5^{(n-3) / 4}<5^{(n-2) / 4}<5^{(n-1) / 4}<5^{n / 4}$, and $5^{(n-i) / 4}=\beta(n-i)$ if $4 \mid(n-i)$ with $i=0,1,2,3$. If $i>0$, then $\beta(n-i)<\beta(n)$ by Lemma 2.1. If $i=0$, then $\beta(n)=5^{n / 4}>5^{(n-3) / 4}$, whence the result.

For use in later sections, we record the following lemma. 
Lemma 2.3. Let $n \in \mathbb{N}$ be even and $n>6$. Set $\beta^{\prime}(n)=\max _{a \text { odd }} \beta(a) \beta(n-a)$. Then we have $\beta^{\prime}(n)=\beta(5) \beta(n-5)$. Explicitly, we have

$$
\beta^{\prime}(n)= \begin{cases}7^{2} \cdot 5^{(n-10) / 4} & \text { if } n \equiv 2 \bmod 4, \\ 7^{2} \cdot 11 \cdot 5^{(n-16) / 4} & \text { if } n \equiv 0 \bmod 4 \text { and } n \geq 16,\end{cases}
$$

and $\beta^{\prime}(8)=7 \cdot 3, \beta^{\prime}(12)=7 \cdot 15$. In addition, $\beta^{\prime}(6)=9, \beta^{\prime}(4)=3, \beta^{\prime}(2)=1$.

Proof. For the additional statement, see Table 2. Suppose that $i, j \in \mathbb{N}$ is odd with $i+j=n, i \leq j$. Since $n \geq 10$, we have $j \geq 5$. If $j \equiv 3 \bmod 4$ and $j>7$, then $\beta(j-5)=\beta(6) \beta(j-11)$ by Theorem 1.2. Hence Theorem 1.2 and Lemma 2.1 imply for $j \neq 7, \beta(i) \beta(j)=\beta(i) \beta(5) \beta(j-5) \leq \beta(5) \beta(n-5)$. For $j=7$ and $i \in\{3,7\}$, we have

$$
\beta(3) \beta(7)=3 \cdot 15<7^{2}=\beta(5)^{2} \quad \text { and } \quad \beta(7)^{2}=15^{2}<7 \cdot 35=\beta(5) \beta(9) .
$$

So, in any case, $\beta^{\prime}(n)=\beta(5) \beta(n-5)$. Hence, applying Theorem 1.2, we obtain the formulae for $\beta^{\prime}(n)$ stated above.

\subsection{Unipotent characters}

Let $\mathbf{G}$ be a connected reductive algebraic group with Frobenius endomorphism $F$. For a precise definition of it, we refer to [3, p.31] or [12, Section 2.1]. (Some authors use the terms "Frobenius map" or "Steinberg endomorphism".) If $\mathbf{G}$ is simple, then an algebraic group endomorphism $F: \mathbf{G} \rightarrow \mathbf{G}$ is Frobenius if and only if the subgroup $\mathbf{G}^{F}=\{g \in \mathbf{G}: F(g)=g\}$ is finite [12, Theorem 21.5]. Groups $\mathbf{G}^{F}$ are called finite reductive groups (see [2, p. XIII] or [3, § 4.4]). (The term "finite groups of Lie type" is also in use in the literature; see [3, p.31].) Thus, a finite reductive group is determined by the pair $(\mathbf{G}, F)$, a connected reductive algebraic group $\mathbf{G}$ and a Frobenius endomorphism $F$ of it.

As briefly mentioned in Section 1, for every finite reductive group $G=\mathbf{G}^{F}$, the Deligne-Lusztig theory partitions the set of irreducible characters of $G$ as a disjoint union of the Lusztig (geometric) series $\mathcal{E}_{S}$, where $s$ runs through a set of representatives of the classes of semisimple elements of $G^{*}$ that are conjugate in $\mathbf{G}^{*}$; see [5, Definition 13.16]. The characters in $\varepsilon_{1}$ (that is, for $s=1$ ) are called unipotent. Note that the geometric series can be further refined to rational series parameterized by the conjugacy classes of semisimple elements in $G^{*}$; if $\mathbf{G}$ has connected center (assumed in this paper), then the geometric and rational series coincide [5, p. 107].

We emphasize that the Lusztig series (and hence unipotent characters) of a finite reductive group cannot be defined in terms of $\mathbf{G}^{F}$ as an abstract group. One 
observes that a given finite group of Lie type can be obtained as $\mathbf{G}^{F}$ from different pairs $\mathbf{G}, F$. A typical example is as follows. Given a pair $(\mathbf{G}, F)$, set $\mathbf{H}$ to be the direct product $\mathbf{G} \times \cdots \times \mathbf{G}$ of $m$ copies of $\mathbf{G}$, and then define a Frobenius endomorphism $F^{\prime}$ of $\mathbf{H}$ as a mapping sending an element $\left(g_{1}, \ldots, g_{m}\right)$ with $g_{1}, \ldots, g_{m} \in \mathbf{G}$ to $\left(F\left(g_{m}\right), g_{1}, \ldots, g_{m-1}\right)$. Then $F^{\prime}\left(g_{1}, \ldots, g_{m}\right)=\left(g_{1}, \ldots, g_{m}\right)$ implies

$$
F\left(g_{m}\right)=g_{1}=g_{2}=\cdots=g_{m},
$$

so $\mathbf{H}^{F^{\prime}}=\left\{(g, \ldots, g): g \in \mathbf{G}^{F}\right\} \cong \mathbf{G}^{F}$. In fact, the general case reduces to the above example; see [3, p.380], where it is stated that one can assume $\mathbf{H}$ to be simple (if so is $\mathbf{G}$ ), that is, $m=1$.

Lemma 2.4 ([5, p. 112]). Let $G=\mathbf{G}^{F}$ be a finite reductive group and $s \in G^{*}$ a semisimple element. Suppose that $C_{\mathbf{G}^{*}}(s)$ is connected. Then $\left|\mathcal{E}_{s}\right|=v\left(C_{G^{*}}(s)\right)$, the number of unipotent characters of $C_{G^{*}}(s)$.

To be rigorous, we emphasize that $C_{G^{*}}(s)=C_{\mathbf{G}^{*}}(s)^{F}$ is a finite reductive group.

Lemma 2.4 reduces the computation of the sizes of Lusztig series to the computation of the number of unipotent characters, and our results in fact give sharp upper bounds for $v\left(C_{G^{*}}(s)\right)$ when $s$ ranges over semisimple elements of $G^{*}$. (Note that, if $C_{\mathbf{G}}(s)$ is not connected, one can extend the notion of a unipotent character so that Lemma 2.4 remains valid; see [5, p. 112]. However, in full generality, the problem of computing sharp upper bounds is more complex.)

For what follows, it is essential to decide whether or not $C_{\mathbf{G}}(s)$ is a connected reductive group if $\mathbf{G}$ is and $s \in \mathbf{G}$ is a semisimple element. There are the following criteria for connectivity.

Lemma 2.5. The group $C_{\mathbf{G}}(s)$ is connected for all semisimple elements $s$ of $\mathbf{G}$ if one of the following holds.

(1) The center of $\mathbf{G}^{*}$ is connected.

(2) $\mathbf{G}$ is semisimple and simply connected.

(3) $\mathbf{G}=\mathrm{SO}(\mathbf{V})$, where $\mathbf{V}$ is an orthogonal space over $\overline{\mathbb{F}}_{q}$, and the multiplicity of the eigenvalue 1 or the eigenvalue -1 of $s$ on $\mathbf{V}$ is at most 1 .

Proof. See [5, Remark 13.15] for (1), and [15, Chapter E, Remark 3.9] for (2). In fact, (2) is a particular case of (1) as the dual group of a simply connected semisimple algebraic group has trivial center; see [3, § 4.4].

(3) If $\operatorname{dim} V$ is even, then the multiplicity of the eigenvalue 1 as well as the eigenvalue -1 of $s$ on $\mathbf{V}$ is known to be even (see Lemma 4.2 below for a proof), 
so (3) follows from [16, Lemma 2.2] in this case. Now suppose that $\operatorname{dim} V$ is odd, so, by our convention, $q$ is odd. Let $\mathbf{V}_{1}$ be the 1-eigenspace of $s$ on $\mathbf{V}$. Then $\operatorname{dim} \mathbf{V}_{1}=1$ (Lemma 4.2), so $C_{\mathbf{G}}(s)$ is contained in the stabilizer of $\mathbf{V}_{1}$ in $\mathbf{G}$. With respect to a suitable basis of $\mathbf{V}$, the latter can be written as

$$
\left\{\operatorname{diag}(\operatorname{det} g, g): g \in \mathrm{O}\left(\mathbf{V}_{1}^{\perp}\right)\right\} \text {. }
$$

Then $C_{\mathbf{G}}(s)$ is contained in the group $\operatorname{diag}\left( \pm 1, C_{\mathrm{O}\left(\mathbf{V}_{1}^{\perp}\right)}\left(s^{\prime}\right)\right)$, where $s^{\prime}$ is the restriction of $s$ to $\mathbf{V}_{1}^{\perp}$. As $\operatorname{dim} \mathbf{V}_{1}^{\perp}$ is even, $C_{\mathrm{O}\left(\mathbf{V}_{1}^{\perp}\right)}\left(s^{\prime}\right)$ is connected by the above and hence is contained in $\mathrm{SO}\left(\mathbf{V}_{1}^{\perp}\right)$ (or see the proof of [16, Lemma 2.1]). Then $C_{\mathbf{G}}(s)=\operatorname{diag}\left(1, C_{\mathrm{O}\left(\mathbf{V}_{1}^{\perp}\right)}\left(s^{\prime}\right)\right)$, whence the result.

Remark. There is an inaccuracy in the statement of [16, Lemma 2.2], where "Let $\mathbf{G}=\operatorname{SO}(\mathbf{V})$ " is to be replaced by "Let $\mathbf{G}=\operatorname{SO}(\mathbf{V})$ if $q$ is odd and $\Omega(\mathbf{V})$ if $q$ is even" with no change of the proof.

Thus, if Lemma 2.5 applies, then $C_{G}(s)$ is a finite reductive group. For the notion of a simply connected semisimple algebraic group, see for instance [12, Definition 9.14] or [3, p. 25]; if $\mathbf{G}$ is of adjoint type, then $G^{*}$ is simply connected. Classical algebraic groups of adjoint and simply connected type can be described in terms of their traditional definition; see [12, Table 9.2] or [3, p. 40].

Lemma 2.6. Let $H=\mathrm{SO}(V)$, where $\operatorname{dim} V$ is even, and let $s \in H$ be a semisimple element.

(1) Suppose that either 1 or -1 is not an eigenvalue of $s$ on $V$. Then $C_{H}(s)$ is a finite reductive group. In particular, this is the case if $q$ is even.

(2) Suppose that neither 1 nor -1 is an eigenvalue of $s$ on $V$. Then $C_{\mathrm{O}(V)}(s) \subset H$.

Proof. (1) Let $\mathbf{V}=V \otimes \overline{\mathbb{F}}_{q}$ be an orthogonal space defined with the same Gram matrix as $V$. It is well known that $\mathbf{G}=\mathrm{SO}(\mathbf{V})$ is an algebraic group and

$$
\mathrm{SO}(V)=\mathrm{SO}(\mathbf{V})^{F} \text { for some Frobenius morphism } F: \mathbf{G} \rightarrow \mathbf{G} .
$$

By [16, Lemma 2.2(2)], the group $C_{\mathbf{G}}(s)$ is connected. As $C_{G}(s)=C_{\mathbf{G}}(s)^{F}$, the claim follows.

(2) See [16, Lemma 2.1].

Let $\mathbf{V}$ be an orthogonal space over $\overline{\mathbb{F}}_{q}$. The group $\mathrm{SO}(\mathbf{V})$ is a simple algebraic group; however, $\mathrm{SO}(\mathbf{V})$ is not simply connected. Slightly abusing notation, we denote the simply connected covering of it by $\operatorname{Spin}(\mathbf{V})$; this is the preimage of $\mathrm{SO}(\mathbf{V})$ in the full spinor group of $\mathbf{V}$. So $\mathrm{Spin}(\mathbf{V})$ is a simply connected 
simple algebraic group, and there is a surjective algebraic group homomorphism $\eta: \operatorname{Spin}(\mathbf{V}) \rightarrow \operatorname{SO}(\mathbf{V})$ (see [2, p. 228]). If $q$ is even, then $\eta$ is an isomorphism of the underlying abstract groups.

Let $h: \mathbf{G} \rightarrow \mathbf{H}$ be a surjective homomorphism of connected algebraic groups with central kernel (that is, an isogeny), defined over $\mathbb{F}_{q}$, and let $F$ be a Frobenius endomorphism of $\mathbf{G}$. If $\operatorname{ker} h$ is $F$-stable, one defines the action of $F$ on $\mathbf{H}$ by $F(h(g))=h(F(g))$ for $g \in \mathbf{G}$. Set $H:=\mathbf{H}^{F}$. With this notation, we have the following result.

Lemma 2.7 ([5, Proposition 13.20]). Let $v(G), v(H)$ be the number of unipotent characters of $G, H$, respectively. Then $v(G)=v(H)$.

For instance, if

$$
G=\mathrm{GL}_{n}(q) \text { and } H=\mathrm{PGL}_{n}(q) \text { or } \quad G=\mathrm{U}_{n}(q) \text { and } H=\mathrm{PU}_{n}(q),
$$

then the lemma applies. Moreover, $v\left(\operatorname{SL}_{n}(q)\right)=v\left(\operatorname{PGL}_{n}(q)\right)$ as (see [3, p. 39])

$$
\operatorname{PSL}_{n}\left(\overline{\mathbb{F}}_{q}\right)^{F}=\operatorname{PGL}_{n}(q) \text { for } n>1 .
$$

Lemma 2.7 allows us to ignore the case where $\mathbf{G}^{*}=\operatorname{Spin}_{2 n+1}\left(\overline{\mathbb{F}}_{q}\right), q$ even. Indeed, in this case, there exists an isogeny $h: \operatorname{Spin}_{2 n+1}\left(\overline{\mathbb{F}}_{q}\right) \rightarrow \mathbf{H}:=\operatorname{Sp}_{2 n}\left(\overline{\mathbb{F}}_{q}\right)$, which also yields an isogeny $C_{\mathbf{G}^{*}}(s) \rightarrow C_{\mathbf{H}}(h(s))$. Therefore, by Lemma 2.7, we have $v\left(C_{G^{*}}(s)\right)=v\left(C_{H}(h(s))\right)$, where $H=\mathbf{H}^{F}=\operatorname{Sp}_{2 n}(q)$. So it suffices to compute the maximum of $v\left(C_{H}(s)\right)$ over semisimple elements $s \in H$.

For $\mathrm{SO}_{2 n}\left(\overline{\mathbb{F}}_{q}\right)$, there are Frobenius endomorphisms for which $\mathrm{SO}_{2 n}\left(\overline{\mathbb{F}}_{q}\right)^{F}$ coincides with $\mathrm{SO}_{2 n}^{+}(q)$ or $\mathrm{SO}_{2 n}^{-}(q)$. (If $n=4$, there is one more type of Frobenius endomorphisms which yields the "triality group" ${ }^{3} D_{4}(q)$; this is not considered in this paper.) Here $\mathrm{SO}_{2 n}^{+}(q)$ and $\mathrm{SO}_{2 n}^{-}(q)$ are special orthogonal groups $\mathrm{SO}(V)$, where $V$ is an orthogonal space of Witt defect 0 and 1 , respectively, $\operatorname{dim} V=2 n$.

There exists a Frobenius endomorphism $F$, say, of $\operatorname{Spin}(\mathbf{V})$ compatible with the natural mapping $\eta$ : $\operatorname{Spin}(\mathbf{V}) \rightarrow \mathrm{SO}(\mathbf{V})$ in the sense that $\eta(F(h))=F(\eta(h))$. Then we set $\operatorname{Spin}(V)=\operatorname{Spin}(\mathbf{V})^{F}$. If $q$ is odd, then $\eta(\operatorname{Spin}(V))=\Omega(V) \neq \operatorname{SO}(V)$. Nonetheless, by Lemma 2.7, we have the following result.

Lemma 2.8. If $q$ is odd, then $v(\operatorname{Spin}(V))=v(\operatorname{SO}(V))$.

Lemma 2.9. Let $\mathbf{G}=\operatorname{Spin}(\mathbf{V})$ and $s \in \mathbf{G}$ a semisimple element. Let $\eta: \mathbf{G} \rightarrow \mathrm{SO}(\mathbf{V})$ be the natural projection. Let $\mathbf{W}_{1}, \mathbf{W}_{2}$ be the 1- and-1-eigenspaces of $\eta(s)$ on $\mathbf{V}$, and $\mathbf{W}_{3}=\left(\mathbf{W}_{1}+\mathbf{W}_{2}\right)^{\perp}$. Then $\eta\left(C_{\mathbf{G}}(s)\right)=\mathrm{SO}\left(\mathbf{W}_{1}\right) \times \mathrm{SO}\left(\mathbf{W}_{2}\right) \times C_{\mathrm{SO}_{(}\left(\mathbf{W}_{3}\right)}\left(s^{\prime}\right)$, where $s^{\prime} \in \mathrm{SO}\left(\mathbf{W}_{3}\right)$ is the restriction of $\eta(s)$ to $\mathbf{W}_{3}$. (If $\mathbf{W}_{1}=0$ or $\mathbf{W}_{2}=0$, then the respective multiple is to be dropped.) 
Proof. Clearly, $\eta\left(C_{\mathbf{G}}(s)\right)$ stabilizes $\mathbf{W}_{1}$ and $\mathbf{W}_{2}$, and hence also $\mathbf{W}_{3}$. It follows that $\eta\left(C_{\mathbf{G}}(s)\right) \subset \mathrm{O}\left(\mathbf{W}_{1}\right) \times \mathrm{O}\left(\mathbf{W}_{2}\right) \times \mathrm{O}\left(\mathbf{W}_{3}\right)$. By Lemma 2.5 and the comments after Lemma 2.6, the group $C_{\mathbf{G}}(s)$ is connected, as well as $\eta\left(C_{\mathbf{G}}(s)\right)$.

Observe first that $\eta\left(C_{\mathbf{G}}(s)\right)$ has finite index in $C_{\mathrm{O}(\mathbf{V})}(\eta(s))$. Indeed, let

$$
M=\{g \in \mathbf{G}:[g, s] \in \operatorname{ker} \eta\}
$$

which coincides with $\{g \in \mathbf{G}:[\eta(g), \eta(s)]=1\}=\eta^{-1}\left(C_{\mathrm{SO}(\mathbf{V})}(\eta(s))\right)$. Then

$$
\eta(M)=C_{\mathrm{SO}(\mathrm{V})}(\eta(s))
$$

As ker $\eta \subseteq Z(\mathbf{G})$, it follows that the mapping $g \rightarrow[g, s](g \in M)$ is a homomorphism $M \rightarrow Z(\mathbf{G})$ whose kernel is $C_{\mathbf{G}}(s)$. The group $Z(\mathbf{G})$ is finite, so $C_{\mathbf{G}}(s)$ has finite index in $M$. So $\eta\left(C_{\mathbf{G}}(s)\right)$ has finite index in $\eta(M)=C_{\mathrm{SO}(\mathbf{V})}(\eta(s))$, and hence in $C_{\mathrm{O}(\mathrm{V})}(\eta(s))$.

Choose a basis $B$, say, of $\mathbf{V}$ such that $B \cap \mathbf{W}_{i}$ is a basis of $\mathbf{W}_{i}$ for $i=1,2,3$. Then, under this basis, the matrix $t$ of $\eta(s)$ on $\mathbf{V}$ is $\operatorname{diag}\left(\mathrm{Id},-\mathrm{Id}, s^{\prime}\right)$. Therefore, $C_{\mathrm{O}(\mathbf{V})}(t) \subset \mathrm{O}\left(\mathbf{W}_{1}\right) \times \mathrm{O}\left(\mathbf{W}_{2}\right) \times C_{\mathrm{O}\left(\mathbf{W}_{3}\right)}\left(s^{\prime}\right)$. Note that $s^{\prime} \in \mathrm{SO}\left(\mathbf{W}_{3}\right)$ as $\operatorname{dim} \mathbf{W}_{2}$ is even (Lemma 4.2).

As \pm 1 are not eigenvalues of $s^{\prime}, C_{\mathrm{O}\left(\mathbf{W}_{3}\right)}\left(s^{\prime}\right)$ is connected (Lemma 2.5). In addition, $\mathrm{SO}\left(\mathbf{W}_{1}\right) \times \mathrm{SO}\left(\mathbf{W}_{2}\right) \times C_{\mathrm{O}\left(\mathbf{W}_{3}\right)}\left(s^{\prime}\right)$ is connected (as so is $\left.C_{\mathrm{O}\left(\mathbf{W}_{3}\right)}\left(s^{\prime}\right)\right)$ and has finite index in $\mathrm{O}\left(\mathbf{W}_{1}\right) \times \mathrm{O}\left(\mathbf{W}_{2}\right) \times C_{\mathrm{O}\left(\mathbf{W}_{3}\right)}\left(s^{\prime}\right)$. So both

$$
\eta\left(C_{\mathbf{G}}(s)\right) \text { and } \mathrm{SO}\left(\mathbf{W}_{1}\right) \times \mathrm{SO}\left(\mathbf{W}_{2}\right) \times C_{\mathrm{SO}\left(\mathbf{W}_{3}\right)}\left(s^{\prime}\right)
$$

are connected subgroups of finite index in $\mathrm{O}\left(\mathbf{W}_{1}\right) \times \mathrm{O}\left(\mathbf{W}_{2}\right) \times C_{\mathrm{O}\left(\mathbf{w}_{3}\right)}\left(s^{\prime}\right)$. As the connected component of the identity in an algebraic group is unique, these groups coincide, as stated.

Lemma 2.9 implies the following result on unipotent characters which is essential in what follows.

Lemma 2.10. Let $G=\operatorname{Spin}(V)$ or $\operatorname{Sp}(V)$, where $V$ is an orthogonal or symplectic space over $\mathbb{F}_{q}$. Let $s \in G$ be a semisimple element, and let $W_{1}, W_{2}$ be the 1 - and -1-eigenspaces of $s$ on $V$. Let $W_{3}=\left(W_{1}+W_{2}\right)^{\perp}$. Then

$$
v\left(C_{G}(s)\right)=v\left(\mathrm{SO}\left(W_{1}\right)\right) \cdot v\left(\mathrm{SO}\left(W_{2}\right)\right) \cdot v\left(C_{\mathrm{SO}\left(W_{3}\right)}\left(s^{\prime}\right)\right),
$$

where $s^{\prime}$ is the restriction of $s$ to $W_{3}$.

Proof. We omit the proof for $\operatorname{Sp}(V)$ as it is straightforward. Let $G=\operatorname{Spin}(V)$. Note that $v\left(C_{\mathrm{SO}\left(W_{3}\right)}\left(s^{\prime}\right)\right)$ is meaningful as $C_{\mathrm{SO}\left(W_{3}\right)}\left(s^{\prime}\right)$ is a finite reductive group 
(Lemma 2.6). We use the notation of Lemma 2.9, assuming that $\mathbf{V}=V \otimes \overline{\mathbb{F}}_{q}$ and that the structure of an orthogonal space on $\mathbf{V}$ is defined by the same Gram matrix as that of $V$. Then $\mathbf{W}_{i}=W_{i} \otimes \overline{\mathbb{F}}_{q}$ for $i=1,2,3$. Let $F$ be the Frobenius endomorphism of $\mathbf{G}$ such that $\mathbf{G}^{F}=G$; we keep $F$ for the Frobenius endomorphisms of $\mathrm{SO}(\mathbf{V}), \mathrm{SO}\left(\mathbf{W}_{i}\right)(i=1,2,3)$ inherited from that of $\mathbf{G}$. By Lemma 2.9, we have $\eta\left(C_{\mathbf{G}}(s)\right)=\mathrm{SO}\left(\mathbf{W}_{1}\right) \times \mathrm{SO}\left(\mathbf{W}_{2}\right) \times C_{\mathrm{SO}\left(\mathbf{W}_{3}\right)}\left(s^{\prime}\right)$. By Lemma 2.7 , we have $v\left(\left(\eta\left(C_{\mathbf{G}}(s)\right)\right)^{F}\right)=v\left(C_{\mathbf{G}}(s)^{F}\right)$, and the left-hand side is equal to

$$
\begin{aligned}
& v\left(\mathrm{SO}\left(\mathbf{W}_{1}\right)^{F} \times \mathrm{SO}\left(\mathbf{W}_{2}\right)^{F} \times C_{\mathrm{SO}\left(\mathbf{W}_{3}\right)}\left(s^{\prime}\right)^{F}\right) \\
& \quad=v\left(\mathrm{SO}\left(W_{1}\right)\right) \cdot v\left(\mathrm{SO}\left(W_{2}\right)\right) \cdot v\left(C_{\mathrm{SO}\left(W_{3}\right)}\left(s^{\prime}\right)\right),
\end{aligned}
$$

as claimed.

\section{Proof of Theorem 1.3}

Here $G^{*} \cong \mathrm{GL}_{n}(q)$ or $\mathrm{U}_{n}(q)$. To simplify notation, we deal below with $G$ in place of $G^{*}$, that is, we choose a semisimple element $s \in G$ and show that the number of unipotent characters in $C_{G}(s)$ does not exceed $\beta(n)$.

For our purpose, we quote the following well-known result; see [3, p. 465].

Lemma 3.1. Let $\mathbf{G}=\mathrm{GL}_{n}\left(\overline{\mathbb{F}}_{q}\right)$ and $G=\mathbf{G}^{F} \cong \mathrm{GL}_{n}(q)$ or $\mathrm{U}_{n}(q)$ (depending on $F)$. Then the number of unipotent characters of $G$ equals $p(n)$, the number of partitions of $n$.

Let $G=\mathrm{GL}_{n}(q), V$ the natural $\mathbb{F}_{q} G$-module, and let $s \in G$ be a semisimple element. We can write $V=\bigoplus V_{i}$, where $V_{i}$ are the homogeneous components for $s$, that is, each $V_{i}$ is a sum of isomorphic $\mathbb{F}_{q}\langle s\rangle$-modules, and distinct $V_{i}, V_{j}$ have no common irreducible constituents. Let $s_{i} \in \mathrm{GL}\left(V_{i}\right)$ be the restriction of $s$ to $V_{i}$. Then $C_{G}(s) \subset \prod_{i} \mathrm{GL}\left(V_{i}\right)$, and $C_{G}(s)=\prod_{i} C_{\mathrm{GL}\left(V_{i}\right)}\left(s_{i}\right)$. Let $d_{i}$ be the dimension of a minimal $\mathbb{F}_{q}\langle s\rangle$-submodule of $V_{i}$. Then $C_{\mathrm{GL}\left(V_{i}\right)}\left(s_{i}\right) \cong \mathrm{GL}_{d_{i}}\left(q^{m_{i}}\right)$, where $m_{i}=\operatorname{dim} V_{i} / d_{i}$. One observes that the decomposition $V=\bigoplus V_{i}$ is unique up to reordering the terms. Let $k$ be the number of terms and $n_{i}=\operatorname{dim} V_{i}$. Then $s$ determines the string $\left(n_{1}, \ldots, n_{k}\right)$ up to reordering of the $n_{1}, \ldots, n_{k}$, which is a partition of $n$, and we denote by $\pi(s)$ the partition $\left(n_{1}, \ldots, n_{k}\right)$. (We can assume $n_{1} \geq \cdots \geq n_{k}$, but we prefer to allow any ordering.) If $s \in \mathrm{U}_{n}(q) \subset \mathrm{GL}_{n}\left(q^{2}\right)$, then $\pi(s)$ is defined as the partition obtained for $s$ in $\mathrm{GL}_{n}\left(q^{2}\right)$. The following lemma is well known.

Lemma 3.2. Let $\mathbf{G}=\mathrm{GL}_{n}\left(\overline{\mathbb{F}}_{q}\right), G=\mathbf{G}^{F} \cong \mathrm{GL}_{n}(q)$, and let $s \in G$ be a semisimple element. Then $C_{G}(s)$ is isomorphic to the direct product of groups $\operatorname{GL}_{d_{i}}\left(q^{m_{i}}\right)$, where $\sum_{i} d_{i} m_{i}=n$. 
The following lemma is also well known, but we give a proof for the reader's convenience and in order to make further discussions more transparent.

Lemma 3.3. Let $\mathbf{G}=\mathrm{GL}_{n}\left(\overline{\mathbb{F}}_{q}\right), G=\mathbf{G}^{F} \cong \mathrm{U}_{n}(q)$, and let $s \in G$ be a semisimple element. Then $C_{G}(s)$ is isomorphic to the direct product of groups $\mathrm{GL}_{d_{i}}\left(q^{2 m_{i}}\right)$ and $\mathrm{U}_{e_{j}}\left(q^{l_{j}}\right)$, where $l_{i}$ is odd and $\sum_{i} 2 d_{i} m_{i}+\sum e_{j} l_{j}=n$.

Proof. Note that each of the sums $\sum_{i} 2 d_{i} m_{i}, \sum e_{j} l_{j}$ can be absent. It is well known that there is an orthogonal decomposition $V=\left(\oplus V_{i}\right) \oplus\left(\oplus V_{j}\right)$, where each $V_{j}$ is a non-degenerate homogeneous component for $s$, and each $V_{i}$ is the sum of two totally isotropic homogeneous components for $s$. Let $H$ be the stabilizer in $G$ of this decomposition, that is,

$$
H=\left\{g \in G: g V_{i}=V_{i}, g V_{j}=V_{j} \text { for each term } V_{i}, V_{j}\right\} .
$$

Let $n_{i}=\operatorname{dim} V_{i}, n_{j}=\operatorname{dim} V_{j}$, and let $H_{i}, H_{j}$ be the restriction of $H$ to $V_{i}, V_{j}$, respectively. Then $H_{j} \cong \mathrm{U}_{n_{j}}(q)$ and $H_{i} \cong \mathrm{GL}_{n_{i} / 2}\left(q^{2}\right)$. Therefore,

$$
n=\sum n_{i}+\sum n_{j} \text { and } H \cong\left(\prod_{i} \mathrm{GL}_{n_{i} / 2}\left(q^{2}\right)\right) \times\left(\prod_{j} \mathrm{U}_{n_{j}}(q)\right) .
$$

Let $s_{i}, s_{j}$ be the restriction of $s$ to $V_{i}, V_{j}$, respectively. Then

$$
s_{i} \in H_{i}, \quad s_{j} \in H_{j} \quad \text { and } \quad C_{G}(s)=\left(\prod_{i} C_{H_{i}}\left(s_{i}\right)\right) \times\left(\prod_{j} C_{H_{j}}\left(s_{j}\right)\right) .
$$

Using the isomorphism $H_{i} \cong \mathrm{GL}_{n_{i} / 2}\left(q^{2}\right)$, we can view a homogeneous component $V_{i}^{\prime}$ of $V_{i}$ as a natural $\mathbb{F}_{q^{2}} \mathrm{GL}_{n_{i} / 2}\left(q^{2}\right)$-module, and then $s_{i}$ is a homogeneous element of $\mathrm{GL}_{n_{i} / 2}\left(q^{2}\right)$, that is, $V_{i}^{\prime}$ is a homogeneous $\mathbb{F}_{q}\left\langle s_{i}\right\rangle$-module. As in Lemma 3.2, $C_{H_{i}}\left(s_{i}\right) \cong \operatorname{GL}_{d_{i}}\left(q^{2 m_{i}}\right)$, where $d_{i} m_{i}=\frac{n_{i}}{2}$. It is also known that $C_{H_{j}}\left(s_{j}\right) \cong \mathrm{U}_{e_{j}}\left(q^{2 l_{j}}\right)$, where $e_{j} l_{j}=n_{j}$ and $l_{j}$ is odd. So the result follows.

Lemma 3.4. Let $G=\mathrm{GL}_{n}(q)$ or $\mathrm{U}_{n}(q)$, and let $s \in G^{*}$ be a semisimple element. Then

$$
\left|\mathcal{E}_{s}\right|=v\left(C_{G}(s)\right) \leq \beta(n) .
$$

Furthermore, suppose that equality holds. Then $\pi(s)=\pi(n)$, where $\pi(n)$ is defined in Theorem 1.2, and if $G=\mathrm{GL}_{n}(q)$, then $|s|$ divides $q-1$, if $G=\mathrm{U}_{n}(q)$, then $|s|$ divides $q+1$.

Proof. If $G=\operatorname{GL}_{n}(q)$, then, by Lemma 3.2,

$$
C_{G}(s) \cong \prod_{i} \operatorname{GL}_{d_{i}}\left(q^{m_{i}}\right), \quad \text { where } \sum_{i} d_{i} m_{i}=n
$$


Recall (Lemma 3.1) that the number of unipotent characters of $\mathrm{GL}_{n}(q)$ equals $p(n)$ and hence does not depend on $q$. So $v\left(C_{G^{*}}(s)\right)=\prod_{i} p\left(d_{i}\right)$. Set $n^{\prime}=\sum d_{i}$. Then $\prod_{i} p\left(d_{i}\right) \leq \beta\left(n^{\prime}\right)$. By Lemma 2.1, $\beta\left(n^{\prime}\right)<\beta(n)$ for $n^{\prime}<n$; if equality holds above, then $n=n^{\prime}$, and hence $m_{i}=1$ for every $i$. So the result follows from Lemma 3.1.

Let $G=\mathrm{U}_{n}(q)$. Then $C_{G}(s)$ is a direct product of groups isomorphic to

$$
\operatorname{GL}_{m_{i}}\left(q^{2 d_{i}}\right), \quad i=1, \ldots, k^{\prime}, \quad \text { and } \quad \mathrm{U}_{l_{j}}\left(q^{f_{j}}\right), \quad j=1, \ldots, k^{\prime \prime},
$$

for some integers $k^{\prime}, k^{\prime \prime} \geq 0$, and $n=\operatorname{dim} V=2 \sum_{i=1}^{k^{\prime}} m_{i} d_{i}+\sum_{j=1}^{k^{\prime \prime}} l_{j} f_{j}$. (Note that $C_{G}(s)$ may be a product of $\mathrm{GL}_{m_{i}}\left(q^{2 d_{i}}\right)$ or $\mathrm{U}_{l_{j}}\left(q^{f_{j}}\right)$ only.) The number of unipotent characters of $\mathrm{GL}_{m_{i}}\left(q^{2 d_{i}}\right)$ equals $p\left(m_{i}\right)$ and that of $\mathrm{U}_{l_{j}}\left(q^{f_{j}}\right)$ equals $p\left(l_{j}\right)$ (Lemma 3.1). Let $n^{\prime}=\sum m_{i}, n^{\prime \prime}=\sum l_{j}$. Then

$$
\left|\mathcal{E}_{s}\right|=v\left(C_{G}(s)\right)=\prod p\left(m_{i}\right) \cdot \prod p\left(l_{j}\right) \leq \beta\left(n^{\prime}\right) \cdot \beta\left(n^{\prime \prime}\right) .
$$

By Lemma 2.1, $\beta\left(n^{\prime}\right) \cdot \beta\left(n^{\prime \prime}\right) \leq \beta\left(n^{\prime}+n^{\prime \prime}\right) \leq \beta(n)$. If the equality holds, then $n=n^{\prime}+n^{\prime \prime}$, whence $n^{\prime}=0, n=n^{\prime \prime}$ and $f_{j}=1$ for all $j=1, \ldots, k^{\prime \prime}$. It follows that $|s|$ divides $q+1$, and $\pi(s)=\pi(n)$ again follows from Lemma 3.1.

We now show that the bound is attained for every $n$ for $q$ large enough.

Lemma 3.5. Let $n, i \in \mathbb{N}, i \in\{0,1,2,3\}$ with $i \equiv n \bmod 4$. Assume that $n>3$ if $i=0,1,2$, and $n>10$ for $i=3$. Let $G=\operatorname{GL}_{n}(q)$, respectively, $\mathrm{U}_{n}(q)$. If $n \leq 4(q-1)+i$, respectively, $n \leq 4(q+1)+i$, then $v\left(C_{G}(s)\right)=\beta(n)$ for $a$ suitable semisimple element $s \in G$.

Proof. Let $n=4 k+i$. Then $k \leq q-1$, respectively, $q+1$. Therefore, there exist distinct elements $a_{1}, \ldots, a_{k} \in \mathrm{GL}_{1}(q)$, respectively, $\mathrm{U}_{1}(q)$. If $i=0$, then we set $s=\operatorname{diag}\left(a_{1} \cdot \mathrm{Id}_{4}, a_{2} \cdot \mathrm{Id}_{4}, \ldots, a_{k} \cdot \operatorname{Id}_{4}\right)$. If $i=1$, then we take the last scalar to be $a_{k} \cdot \operatorname{Id}_{5}$; if $i=2$, then we take the last scalar to be $a_{k} \cdot \operatorname{Id}_{6}$. If $i=3$, then we take the last two scalars to be $a_{k-1} \cdot \mathrm{Id}_{5}$ and $a_{k} \cdot \operatorname{Id}_{6}$. If $G=\mathrm{U}_{n}(q)$, then we choose an orthogonal basis of the underlying space in order to get $s \in \mathrm{U}_{n}(q)$. Then $C_{G}(s)$ is the direct product of groups $\mathrm{GL}_{4}(q)$ (respectively $\left.\mathrm{U}_{4}(q)\right)$ if $n \equiv 0 \bmod 4$, with obvious adjustments in the other cases. Then $v\left(C_{G^{*}}(s)\right)=\beta(n)$. So the bound $\beta(n)$ is attained.

Proof of Theorem 1.3. This follows from Lemmas 3.5 and 3.4.

Lemma 3.6. Let $C$ be a cyclic group, $|C|>2$. Set $l=\frac{|C|-2}{2}$ if $|C|$ is even, and $l=\frac{|C|-1}{2}$ if $|C|$ is odd. Then there are $l$ distinct elements $a_{1}, \ldots, a_{l} \in C$ such that $a_{i} a_{j} \neq 1$ for all $1 \leq i, j \leq l$. 
Proof. Let $C=\langle a\rangle$. Then set $a_{i}=a^{i}$. As the elements $a^{i}(1 \leq i \leq|C|-1)$ are all distinct and $a^{l+1}$ is of order 2 if $|C|$ is even, it follows that $\left\{a^{j}: 1 \leq j \leq l\right\}$ satisfies the conclusion of the lemma.

For application to other classical groups, we need a slightly different version of Lemma 3.5. We view $\mathrm{GL}_{n}(q)$ as a matrix group over $\mathbb{F}_{q}$ and $\mathrm{U}_{n}(q)$ as a matrix group over $\mathbb{F}_{q^{2}}$ whose subgroup of diagonal matrices is $\operatorname{diag}\left(\mathrm{U}_{1}(q), \ldots, \mathrm{U}_{1}(q)\right)$. In Lemma 3.7 below, $D$ denotes the group of diagonal matrices in $G$. For $d \in D$, the set of distinct diagonal entries of $d$ is denoted by $\operatorname{Spec}(d)$.

Lemma 3.7. Let $G=\mathrm{GL}_{n}(q)$ or $\mathrm{U}_{n}(q)$, and let $G_{2}$ be the subgroup of $G$ of index 2 if $q$ is odd, and $G_{2}=G$ if $q$ is even. Suppose that $q \geq n+5$. Then there exists a semisimple element $s \in D \cap G_{2}$ such that

$$
\operatorname{Spec}(s) \cap \operatorname{Spec}\left(s^{-1}\right)=\emptyset \quad \text { and } \quad v\left(C_{G}(s)\right)=\beta(n) .
$$

Proof. Let $C=\mathrm{GL}_{1}(q)$ or $\mathrm{U}_{1}(q)$ if $q$ is even, and let $C$ be the subgroup of index 2 in these groups if $q$ is odd. Let $l$ be as in Lemma 3.6. Then $l=\frac{q-2}{2}$ if $q$ is even, $\frac{q-3}{2}$ if $q \equiv 3 \bmod 4$ and $\frac{q-5}{2}$ if $q \equiv 1 \bmod 4$. By Lemma 3.6, for every $k \leq l$, there are distinct elements $a_{1}, \ldots, a_{k} \in C$ such that $a_{i} a_{j} \neq 1$ for all $1 \leq i, j \leq k$.

Then we take $k=\frac{n-r}{4}$, where $0 \leq r<4$ and $n \equiv r \bmod 4$. As $q \geq n+5$ by assumption, we have $k \stackrel{4}{=} \frac{n-r}{4} \leq \frac{q-5}{4} \leq l$. Let us choose these elements $a_{1}, \ldots, a_{k}$ for a similar reasoning as in the proof of Lemma 3.5 to construct suitable elements $s \in D$.

Then $v\left(C_{G}(s)\right)=\beta(n)$ by Lemma 3.1. In addition, as $s$ is a diagonal matrix with entries $a_{1}, \ldots, a_{k}$ (with certain multiplicities), the condition $a_{i} a_{j} \neq 1$ for all $1 \leq i, j \leq k$ implies $\operatorname{Spec}(s) \cap \operatorname{Spec}\left(s^{-1}\right)=\emptyset$. As each diagonal entry of $s$ lies in $C$, it follows that $s \in G_{2}$.

\section{Other classical groups}

\subsection{Remarks on classical groups}

We start with observations on the centralizers of semisimple elements of classical groups. Let

$$
H \in\left\{\mathrm{O}_{2 n+1}(q), q \text { odd, } \operatorname{Sp}_{2 n}(q), \mathrm{O}_{2 n}^{ \pm}(q)\right\},
$$

and let $V$ be the underlying space for $H$. Recall that $\Omega_{2 n}^{ \pm}(q)$ denotes the subgroup of $\mathrm{O}_{2 n}^{ \pm}(q)$ formed by elements of spinor norm 1 , and in even characteristic $\Omega_{2 n}^{ \pm}(q)=\mathrm{SO}_{2 n}^{ \pm}(q)$ by convention.

The following two lemmas are well known. 
Lemma 4.1 ([8, Proposition 2.5.13]). For $q$ odd, set $\varepsilon(n)=(-1)^{(q-1) n / 2}$. The group $\Omega_{2 n}^{+}(q)$, respectively, $\Omega_{2 n}^{-}(q)$ contains - Id if and only if $\varepsilon(n)=1$, respectively, $\varepsilon(n)=-1$. In particular, $\Omega_{2 n}^{+}(q)$ contains $-\operatorname{Id}$ if $n$ is even or $q$ is a square.

Lemma 4.2. Let $G \in\left\{\mathrm{SO}_{2 n+1}(q), q\right.$ odd, $\left.\mathrm{SO}_{2 n}^{ \pm}(q), \mathrm{Sp}_{2 n}(q)\right\}$, and let $V$ be the natural module for $G$. Let $g \in G$ be a semisimple element, and let $V_{1}$ and $V_{2}$ be the 1- and-1-eigenspaces of $g$ on $V$. (If $q$ is even, then $V_{2}=0$ by convention.) Then

(1) $V_{1}$ and $V_{2}$ are non-degenerate and orthogonal to each other;

(2) $\operatorname{dim} V_{2}$ and $\operatorname{dim}\left(V_{1}+V_{2}\right)^{\perp}$ are even;

(3) $\operatorname{dim} V_{1}$ is even unless $G=\mathrm{SO}_{2 n+1}(q)$, in which case $\operatorname{dim} V_{1}$ is odd.

Proof. Let $i \in\{1,2\}$. (1) If $V_{i}$ is degenerate, then $U:=V_{i} \cap V_{i}^{\perp} \neq 0$ is totally isotropic. Let $0 \neq u \in U$, so $\operatorname{dim} u^{\perp}=\operatorname{dim} V-1$ [8, Lemma 2.1.5]. As $g$ is semisimple, $u^{\perp}$ has a $g$-invariant complement $U^{\prime}$, say. Let $v \in U^{\prime}$, and let $f$ be the form on $V$ defining $G$. Then we have $0 \neq f(u, v)=f(g u, g v)=a f(u, g v)$, where $a=1$ or -1 . It follows that $g v=a v$, which is a contradiction as such a $v$ must be in $V_{i}$.

If $V_{1}, V_{2} \neq 0$, then $q$ is odd; choose $0 \neq v_{i} \in V_{i}$; then

$$
f\left(v_{1}, v_{2}\right)=f\left(g v_{1}, g v_{2}\right)=-f\left(v_{1}, v_{2}\right),
$$

whence $f\left(v_{1}, v_{2}\right)=0$.

(2) It suffices to prove this statement for the respective groups over $\overline{\mathbb{F}}_{q}$; in this case, $V$ is the sum of the eigenspaces of $g$, and \pm 1 are not eigenvalues of $g$ on $W:=\left(V_{1}+V_{2}\right)^{\perp}$. Let $e$ be an eigenvalue of $g$ on $W$, so $e \neq \pm 1$, and let $W_{e}$ be the respective eigenspace. Then, for $0 \neq w \in W_{e}$, we have

$$
f(w, w)=f(g w, g w)=e^{2} f(w, w)=0
$$

as $e^{2} \neq 1$. One easily observes that $w^{\perp}=\langle w\rangle+W^{\prime}$, where $W^{\prime}$ is a $g$-stable nondegenerate subspace of $w^{\perp}$. By induction, $\operatorname{dim} W^{\prime}$ is even, and hence so is $\operatorname{dim} W$.

Moreover, if $v \notin w^{\perp}$, then, as in the proof of (1), $g v=e^{-1} v+x$ for $x \in w^{\perp}$. This implies by induction that the determinant of $g_{W}$, the restriction of $g$ to $W$, equals 1 . As $\operatorname{det} g=1$ and $g$ acts on $V_{2}$ as -Id, it follows that $\operatorname{dim} V_{2}$ is even, as claimed.

(3) is obvious as $\operatorname{dim} V_{1}=\operatorname{dim} V-\operatorname{dim} V_{2}-\operatorname{dim} W$.

Next we describe the structure of centralizers of semisimple elements in $H$. This is treated in $[6, \S 1]$ and elsewhere, but we choose to briefly recall the main facts in a form compatible with what follows. 
Let $h \in H$ be a semisimple element. Viewing $V$ as $\langle h\rangle$-space, we can write $V=V_{1} \oplus \cdots \oplus V_{k} \oplus V_{k+1} \oplus \cdots \oplus V_{k+l}$, where $V_{1}, \ldots, V_{k+l}$ are homogeneous components of $V$ for $\langle h\rangle$. (In other words, $V_{1}, \ldots, V_{k+l}$ are $h$-stable; for every $i \in\{1, \ldots, k+l\}$, all irreducible constituents of $\left.V_{i}\right|_{\langle h\rangle}$ are isomorphic to each other and not isomorphic to those of $\left.V_{j}\right|_{\langle h\rangle}$ for every $j \neq i$.) Furthermore, each $V_{i}$ is either non-degenerate or totally isotropic; see for instance [13, Lemma 3.3]. By reordering the terms, we assume that $V_{1}, \ldots, V_{k}$ are totally isotropic (unless $k=0$ ), whereas $V_{k+1}, \ldots, V_{k+l}$ are non-degenerate (unless $l=0$ ). In the former case, for every $i \leq k$, there is another totally isotropic homogeneous component $V_{j}$, say, such that $\left.V_{i}\right|_{\langle h\rangle}$ and $\left.V_{j}\right|_{\langle h\rangle}$ are dual to each other and $V_{i}+V_{j}$ is non-degenerate [13, Lemma 3.3]. It follows that $k=2 m$ is even. We can reorder $V_{1}, \ldots, V_{k}$ so that $V_{i}, V_{k-i+1}$ are dual as $\langle h\rangle$-modules, $i=1, \ldots, m$. Set $h_{i}=\left.h\right|_{V_{i}}$ and $n_{i}=\operatorname{dim} V_{i}$ for $i=1, \ldots, k+l$. If $h_{i}= \pm \mathrm{Id}$, then $V_{i}$ is nondegenerate (Lemma 4.2), and hence $i>k$ in this case.

For $i \in\{1, \ldots, k\}$, set $H_{i}=\mathrm{GL}\left(V_{i}\right)$, and for $i \in\{k+1, \ldots, l\}$, set

$$
H_{i}=\left\{g \in H:\left.g\right|_{V_{j}}=\mathrm{Id} \text { whenever } j \neq i\right\} \cong I\left(V_{i}\right) .
$$

(For uniformity, we use $I\left(V_{i}\right)$ to denote the classical groups defined by the relevant form on $V_{i}$.) Then

$$
C_{H}(h) \cong C_{H_{1}}\left(h_{1}\right) \times \cdots \times C_{H_{m}}\left(h_{m}\right) \times C_{H_{k+1}}\left(h_{k+1}\right) \times \cdots \times C_{H_{k+l}}\left(h_{k+l}\right) .
$$

Let $d_{i}$ be the dimension of each irreducible constituent of $h_{i}, i=1, \ldots, k+l$. As $V_{i}$ is homogeneous, $n_{i}$ is a multiple of $d_{i}$. Write $n_{i}=d_{i} e_{i}$. If $i \leq k$, then $C_{H_{i}}\left(h_{i}\right)=C_{\mathrm{GL}\left(V_{i}\right)}\left(h_{i}\right)$.

(a) Suppose that $H$ is symplectic. Then

$$
C_{H_{i}}\left(h_{i}\right) \cong \begin{cases}\operatorname{Sp}_{n_{i}}(q) & \text { if } h_{i}= \pm \mathrm{Id}, \\ \operatorname{GL}_{e_{i}}\left(q^{d_{i}}\right) & \text { if } i \leq k, \\ \mathrm{U}_{e_{i}}\left(q^{d_{i} / 2}\right) & \text { if } i>k \text { and } h_{i} \neq \pm \mathrm{Id} .\end{cases}
$$

Here we write $\mathrm{U}_{e_{i}}\left(q^{d_{i} / 2}\right)$ due to our notation for unitary groups, that is,

$$
\mathrm{U}_{e_{i}}\left(q^{d_{i} / 2}\right) \subset \operatorname{GL}_{e_{i}}\left(q^{d_{i}}\right) .
$$

(b) Suppose that $H$ is orthogonal. Then

$$
C_{H_{i}}\left(h_{i}\right) \cong \begin{cases}\mathrm{O}\left(V_{i}\right) & \text { if } h_{i}= \pm \mathrm{Id}, \\ \mathrm{GL}_{e_{i}}\left(q^{d_{i}}\right) & \text { if } h_{i} \neq \pm \mathrm{Id} \text { and } i \leq k, \\ \mathrm{U}_{e_{i}}\left(q^{d_{i} / 2}\right) & \text { if } h_{i} \neq \pm \mathrm{Id} \text { and } i>k, \text { where } e_{i} \text { is odd } \\ & \text { if and only if the Witt defect of } V_{i} \text { is } 1 .\end{cases}
$$


In case (b), fix some $V_{i}$ of Witt defect 1 (assuming the existence of it). Then $V_{i}$ is a direct sum of $e_{i}$ irreducible non-degenerate $\left\langle h_{i}\right\rangle$-modules isomorphic to each other. Denote by $D$ one of them, so $h_{i}$ acts irreducibly on $D$. Here $\operatorname{dim} D>1$ as $h_{i} \neq \pm 1$. Therefore, the Witt defect of $D$ is 1 because otherwise $\mathrm{O}(D)$ has no irreducible element [7, Satz 3 (c)]. So the assertion on the parity of $e_{i}$ follows from [8, Proposition 2.5.11 (ii)]. (Note that $d_{i}=\frac{1}{2} \operatorname{dim} D$ can be even.)

We state the above information in a uniform way as follows.

Proposition 4.3. Let $h \in H$ be a semisimple element, and let $V_{1}, V_{2}$ be the 1 - and -1 -eigenspace of $h$ on $V$. Then

$$
C_{H}(h) \cong I\left(V_{1}\right) \times I\left(V_{2}\right) \times \prod_{i} \mathrm{GL}_{d_{i}}\left(q^{l_{i}}\right) \times \prod_{j} \mathrm{U}_{e_{j}}\left(q^{m_{j}}\right),
$$

where $\frac{1}{2}\left(\operatorname{dim} V_{1}+\operatorname{dim} V_{2}\right)+\sum_{i} d_{i} l_{i}+\sum e_{j} m_{j}=n$.

Corollary 4.4. Let $G \in\left\{\mathrm{SO}_{2 n+1}(q), q\right.$ odd, $\left.\mathrm{SO}_{2 n}^{ \pm}(q), \mathrm{Sp}_{2 n}(q)\right\}$, and let $V$ be the natural module for $G$. Let $s \in G$ be a semisimple element. Suppose that $s$ does not have eigenvalues -1 on $V$ and the multiplicity of the eigenvalue 1 is at most 1 . Then $C_{G}(s) \cong \prod_{i} \mathrm{GL}_{d_{i}}\left(q^{l_{i}}\right) \times \prod_{j} \mathrm{U}_{e_{j}}\left(q^{m_{j}}\right)$, where $\sum_{i} d_{i} l_{i}+\sum e_{j} m_{j}=n$.

Proof. Let $H=I(V)$, so $G \subseteq H$. Suppose that $\operatorname{dim} V$ is even. Then, under these assumptions, $C_{G}(s)=C_{H}(s)$ by Lemma $2.6(2)$, so the result follows from Proposition 4.3. If $\operatorname{dim} V$ is odd, then

$$
\mathrm{O}(V)=\mathrm{SO}(V) \times\{ \pm \mathrm{Id}\}
$$

so $C_{H}(s)=C_{G}(s) \times\{ \pm \mathrm{Id}\}$.

Lemma 4.5. Let $s \in G=\mathrm{SO}_{2 n}^{-}(q)$ be a homogeneous semisimple element, and $s \neq \pm \mathrm{Id}$. Then $C_{G}(s) \cong \mathrm{U}_{e}\left(q^{d}\right)$, where ed $=n$, e is odd, and $v\left(C_{G}(s)\right) \leq p\left(n^{\prime}\right)$, where $n^{\prime}$ is the greatest odd divisor of $n$. In addition, if $\left(n^{\prime}, q\right) \neq(n, 3)$, there exists a (homogeneous) semisimple element $s^{\prime} \in \Omega_{2 n}^{-}(q)$ such that $C_{G}\left(s^{\prime}\right) \cong \mathrm{U}_{n^{\prime}}\left(q^{n / n^{\prime}}\right)$.

Proof. By the comment prior to Proposition 4.3 and Lemma 2.6(2), we have $C_{G}(s) \cong \mathrm{U}_{e}\left(q^{d}\right)$, where $e$ is odd and $n=d e$. By Lemma 3.1, $v\left(\mathrm{U}_{e}\left(q^{d}\right)\right)=p(e)$, and $p(e) \leq p\left(n^{\prime}\right)$. For the additional claim, decompose the natural $\mathbb{F}_{q} G$-module $V$ as a direct sum of $n^{\prime}$ non-degenerate subspaces of dimension $\frac{2 n}{n^{\prime}}$ and of Witt defect 1 . Let $D$ be one of them. Then $\mathrm{SO}(D) \cong \mathrm{SO}_{2 n / n^{\prime}}^{-}(q)$, so $\mathrm{SO}(D)$ contains an irreducible element $t$, say, of order $q^{n / n^{\prime}}+1$ (see [7]). Then $t^{2}$ is still irreducible on $D$ unless $n=n^{\prime}$ and $q=3$. Choose $s$ to be an element of $G$ stabilizing each direct summand (which is isomorphic to $D$ ) and acting on each of them as $t^{2}$ does. Then $s$ is homogeneous and $C_{G}(s) \cong \mathrm{U}_{n^{\prime}}\left(q^{n / n^{\prime}}\right)$ by the above. So the claim follows. 


\subsection{Subgroups of classical groups and their unipotent characters}

We assume the group $\mathbf{G}^{*}$ to be simply connected, which in turn guarantees $C_{\mathbf{G}^{*}}(s)$ to be connected for every semisimple element $s \in \mathbf{G}^{*}$; see Lemma 2.5 (2). In view of Lemma 2.4, our task is to obtain a sharp upper bound for $v\left(C_{G^{*}}(s)\right)$. The information on the number of unipotent characters of $G$ is given in [3, Section 13.8].

If $G^{*}=\operatorname{Spin}_{2 n+1}(q), q$ odd, or $\operatorname{Spin}_{2 n}^{ \pm}(q)$, then the natural module $V$, say, for $\mathrm{O}_{2 n+1}(q)$ or $\mathrm{O}_{2 n}^{ \pm}(q)$ can be viewed as $\mathbb{F}_{q} G^{*}$-module under the natural homomorphism of $G^{*}$ into the respective classical group. So we refer to $V$ as the natural module for $G^{*}$.

We have seen that the function $\beta(n)$ plays a significant role in this paper. It is not true that $\left|\mathcal{E}_{S}\right| \leq \beta(n)$, but the following lemma singles out an important special case where this is true.

Lemma 4.6. Let $G^{*} \in\left\{\operatorname{Spin}_{2 n+1}(q)\right.$ for $q$ odd, $\left.\operatorname{Spin}_{2 n}^{ \pm}(q), \operatorname{Sp}_{2 n}(q)\right\}$, and let $V$ be the natural module for $G^{*}$. Let $s \in G^{*}$ be a semisimple element such that the multiplicity of eigenvalues 1 and -1 of $s$ on $V$ does not exceed 1.

(1) $\left|\varepsilon_{S}\right|=v\left(C_{G^{*}}(s)\right) \leq \beta(n)$.

(2) If $V=V^{\prime} \oplus V^{\prime \prime}$ is an orthogonal decomposition such that

$$
s V^{\prime}=V^{\prime}, \quad s V^{\prime \prime}=V^{\prime \prime} \text { and } \operatorname{Hom}_{s}\left(V^{\prime}, V^{\prime \prime}\right)=0
$$

(equivalently, s has no common eigenvalue on $\mathbf{V}^{\prime}, \mathbf{V}^{\prime \prime}$ ), then

$$
v\left(C_{G^{*}}(s)\right)=v\left(C_{\mathrm{SO}\left(V^{\prime}\right)}\left(s_{1}\right)\right) \cdot v\left(C_{\mathrm{SO}\left(V^{\prime \prime}\right)}\left(s_{2}\right)\right),
$$

where $s_{1}, s_{2}$ are the restriction of $s$ to $V^{\prime}, V^{\prime \prime}$, respectively.

Proof. By Lemma 4.2, the multiplicity of the eigenvalue -1 is always even, as is that of the eigenvalue 1 unless $G^{*} \cong \operatorname{Spin}_{2 n+1}(q)$, where the multiplicity of the eigenvalue 1 is always odd. Therefore, the assumption implies that -1 is not an eigenvalue of $s$, as well as 1 , provided $G^{*} \neq \operatorname{Spin}_{2 n+1}(q)$. By Lemma 2.5, $C_{\mathbf{G}^{*}}(s)$ is connected, so by Lemma $2.4,\left|\varepsilon_{S}\right|=v\left(C_{G^{*}}(s)\right)$.

(1) Let $\mathbf{H} \in\left\{\mathrm{SO}_{2 n+1}\left(\overline{\mathbb{F}}_{q}\right), q\right.$ odd, $\left.\mathrm{SO}_{2 n}\left(\overline{\mathbb{F}}_{q}\right), \mathrm{Sp}_{2 n}\left(\overline{\mathbb{F}}_{q}\right)\right\}$, and $\eta: \mathbf{G}^{*} \rightarrow \mathbf{H}$ the natural homomorphism. Keep $F$ to denote the Frobenius endomorphism of $\mathbf{H}$ inherited from that of $\mathbf{G}^{*}$, and set $H=\mathbf{H}^{F}$. Then $\eta$ is surjective, and $H$ is one of the groups $\mathrm{SO}_{2 n+1}(q), q$ odd, $\mathrm{SO}_{2 n}^{ \pm}(q), \mathrm{Sp}_{2 n}(q)$ (depending on $G^{*}$ and $F$ ). As -1 is not an eigenvalue of $\eta(s)$, by Lemma $2.5(3)$, the group $C_{\mathbf{H}}(\eta(s))$ is connected and, by Lemma 2.6, $v\left(C_{G^{*}}(s)\right)=v\left(C_{H}(\eta(s))\right)$. By Corollary 4.4, we have $C_{H}(s) \cong \prod_{i} \mathrm{GL}_{d_{i}}\left(q^{l_{i}}\right) \times \prod_{j} \mathrm{U}_{e_{j}}\left(q^{m_{j}}\right)$, where $\sum_{i} d_{i} l_{i}+\sum e_{j} m_{j}=n$. Furthermore, the number of unipotent characters of each factor is equal to $p\left(d_{i}\right)$ or $p\left(e_{j}\right)$ 
(Lemma 3.1), so the total is $\prod_{i} p\left(d_{i}\right) \cdot \prod_{j} p\left(e_{j}\right)$. By [1], this number is not greater than $\beta(n)$, whence (1).

(2) By Lemma $2.5(3)$, the group $C_{\mathrm{O}\left(\mathrm{V}_{i}\right)}\left(s_{i}\right)=C_{\mathrm{SO}\left(\mathrm{v}_{i}\right)}\left(s_{i}\right)$ is connected for $i=1,2$, so $C_{\mathrm{O}(\mathrm{V})}(s)=C_{\mathrm{SO}(\mathrm{V})}(s)=C_{\mathrm{SO}\left(\mathrm{V}_{1}\right)}\left(s_{1}\right) \times C_{\mathrm{SO}\left(\mathrm{V}_{2}\right)}\left(s_{2}\right)$. In addition,

$$
C_{\mathrm{SO}\left(\mathrm{v}_{i}\right)}\left(s_{i}\right)^{F}=C_{\mathrm{SO}\left(V_{i}\right)}\left(s_{i}\right),
$$

so $C_{\mathrm{SO}(V)}(s)=C_{\mathrm{SO}\left(V_{1}\right)}\left(s_{1}\right) \times C_{\mathrm{SO}\left(V_{2}\right)}\left(s_{2}\right)$. This implies (2).

The following lemma tells us that the bound in Lemma 4.6(1) is attained if $q$ is large enough and $G^{*} \neq \operatorname{Spin}_{2 n}^{-}(q)$.

Lemma 4.7. Let $G^{*} \in\left\{\operatorname{Spin}_{2 n+1}(q), q\right.$ odd, $\left.\operatorname{Sp}_{2 n}(q), \operatorname{Spin}_{2 n}^{+}(q)\right\}$, and let $V$ be the natural $\mathbb{F}_{q} G^{*}$-module. Suppose that $n \leq q-5$. Then there exists $t \in G^{*}$ such that $V$ is the sum of the eigenspaces of $t$, the multiplicity of the eigenvalues 1 and -1 of $t$ is at most 1 and $v\left(C_{G^{*}}(t)\right)=\beta(n)$. In addition, if $q$ is odd and $G^{*}$ is orthogonal, then $t$ can be chosen in a subgroup of index 2 of $G^{*}$.

Proof. It is well known that there exist totally singular subspaces $V_{1}, V_{2}$ of $V$ such that $V_{1} \cap V_{2}=0, \operatorname{dim} V_{1}=\operatorname{dim} V_{2}=n, V_{1}+V_{2}$ is non-degenerate, and there are dual bases in $V_{1}, V_{2}$ in the sense that if $g \in G$ with $g V_{i}=V_{i}(i=1,2)$ and $g_{i}$ is the matrix of $g$ on $V_{i}$, then $g_{2}={ }^{T} g_{1}^{-1}$, where ${ }^{T} g_{1}$ is the transpose of $g_{1}$. Moreover, for $H=\mathrm{GL}\left(V_{1}\right) \cong \mathrm{GL}_{n}(q)$, there is an embedding $\lambda: H \rightarrow G$ such that $\lambda(h)=\operatorname{diag}\left(h,{ }^{T} h^{-1}\right)$ or $\operatorname{diag}\left(h, 1,{ }^{T} h^{-1}\right)$ for $h \in H$. Let $W$ be the natural module for $H$. It follows that $\left.V_{1}\right|_{H} \cong W$ and $\left.V_{2}\right|_{H}$ is dual to $W$.

Let $s \in H$ be as in Lemma 3.7 and $t=\lambda(s)$. Then the statement on the eigenvalues of $t$ on $V$ is obvious. Since $W$ is the sum of the eigenspaces of $s$, it follows that $V$ is the sum of the eigenspaces of $t$. In addition, the choice of $s$ in Lemma 3.7 implies every eigenspace of $\lambda(s)$ to lie in $V_{1}$ or $V_{2}$. It easily follows that $C_{G^{*}}(t)=\lambda\left(C_{H}(s)\right) \cong C_{H}(s)$. Therefore, the number of unipotent characters of $C_{G^{*}}(t)$ and $C_{H}(s)$ is the same. By Lemma 3.7, the latter is equal to $\beta(n)$, whence the result.

However, to be precise, the isomorphism $C_{H}(s) \rightarrow C_{G^{*}}(t)$ should be accompanied with an isomorphism of algebraic groups $C_{\mathbf{H}}(s) \rightarrow C_{\mathbf{G}^{*}}(t)$ such that

$$
C_{\mathbf{H}}(s)^{F}=C_{H}(s) \text { and } C_{\mathbf{G}^{*}}(t)^{F}=C_{G^{*}}(t) .
$$

(As above, we use the same letter $F$ for the Frobenius endomorphism of different groups $C_{\mathbf{H}}(s)$ and $\left.C_{\mathbf{G}^{*}}(t)\right)$.

Let $\mathbf{G}^{*}=\operatorname{Spin}_{2 n+1}\left(\overline{\mathbb{F}_{q}}\right), \operatorname{Sp}_{2 n}\left(\overline{\mathbb{F}}_{q}\right)$, or $\operatorname{Spin}_{2 n}\left(\overline{\mathbb{F}}_{q}\right)$ and $\mathbf{H}=\mathrm{GL}_{n}\left(\overline{\mathbb{F}}_{q}\right)$. In each case, we choose for $F$ the standard Frobenius endomorphism arising from raising matrix entries of elements of the above groups to the $q$-power (see [5, p. 37]). (For 
this, we choose a basis $B$ in $V$ as above, such that $B \cap V_{1}$ and $B \cap V_{2}$ are dual bases, and view it as a basis of the underlying space $\mathbf{V}$ of $\mathbf{G}$.) Then $G^{*}=\mathbf{G}^{* F}$ and $H=\mathbf{H}^{F}$. The latter holds true when we consider $\mathbf{H}$ as $\mathrm{GL}\left(\mathbf{V}_{1}\right)$ or as a subgroup of $\mathbf{G}^{*}$ stabilizing $\mathbf{V}_{1}$ and $\mathbf{V}_{2}$. Then $C_{\mathbf{H}}(s)$ and $C_{\mathbf{G}^{*}}(t)$ are isomorphic as the eigenvalues of $s$ on $\mathbf{V}_{2}$ are the inverses of those on $\mathbf{V}_{1}$ (see Lemma 3.7). In addition, we have $C_{\mathbf{H}}(s)^{F}=C_{H}(s)$ and $C_{\mathbf{G}^{*}}(t)^{F}=C_{G^{*}}(t)$.

For the additional statement for $q$ odd, let $G_{2}$ be the subgroup of index 2 in $G^{*}$. Then $\left|\lambda(H):\left(\lambda(H) \cap G_{2}\right)\right| \leq 2$. By Lemma 3.7, $s$ can be chosen in the subgroup of index 2 in $H$, whence the claim.

Now we consider the case where $G^{*}=\operatorname{Spin}_{2 n}^{-}(q)$. We shall see that the statement of Lemma 4.7 remains true for $n$ odd but fails otherwise. Recall that

$$
\beta^{\prime}(n)=\max _{a \text { odd }} \beta(a) \beta(n-a)
$$

by Lemma 2.3, $\beta^{\prime}(n)=\beta(5) \beta(n-5)$ for $n>6$.

Lemma 4.8. Let $G^{*}=\operatorname{Spin}_{2 n}^{-}(q)$, and let $V$ be the natural module for $G^{*}$. Let $s \in G^{*}$ be a semisimple element such that 1 and -1 are not eigenvalues of $s$ on $V$.

(1) $v\left(C_{G^{*}}(s)\right) \leq \beta(n)$ for $n$ odd, and $v\left(C_{G^{*}}(s)\right) \leq \beta(5) \beta(n-5)$ for $n>6$ even.

(2) Suppose that $q \geq n+5$. Then the bounds in (1) are attained for some $s$.

(3) If $n=6,4,2$, then the maximum of $v\left(C_{G^{*}}(s)\right)$ equals $9,3,2$, respectively.

Proof. Let $\eta: G^{*} \rightarrow \mathrm{SO}(V)$ be the natural homomorphism and

$$
t=\eta(s) \in \Omega(V) \cong \Omega_{2 n}^{-}(q) .
$$

As observed in Lemma 4.6, $v\left(C_{G^{*}}(s)\right)=v\left(C_{\mathrm{SO}(V)}(t)\right)$.

If $n \leq 7$, the claims follow by inspection, whence (3). Suppose that $n>7$.

(2) Let $V=V_{1} \oplus V_{2}$, where $V_{1}, V_{2}$ are non-degenerate subspaces of $V$, the Witt defect of $V_{1}$ is 1 , the Witt defect of $V_{2}$ is 0 , and $\operatorname{dim} V_{1}=10, \operatorname{dim} V_{2}=2 n-10$. By Lemma 4.7, there is an element $s_{2} \in \Omega\left(V_{2}\right) \cong \Omega_{2 n-10}^{+}(q)$ such that $V_{2}$ is the sum of eigenspaces of $s_{2}$ (whence $\left.s_{2}^{q-1}=1\right)$ and $v\left(C_{\mathrm{SO}\left(V_{2}\right)}\left(s_{2}\right)\right)=\beta(n-5)$.

Furthermore, there is a homogeneous element $s_{1} \in \Omega\left(V_{1}\right) \cong \Omega_{10}^{-}(q)$ such that $\left|s_{1}\right|>2$ divides $q+1$ and $C_{\mathrm{SO}\left(V_{1}\right)}\left(s_{1}\right) \cong \mathrm{U}_{5}(q)$ (see Lemma 4.5 and its proof). As $s_{2}^{q-1}=1$, it follows that $s_{1}, s_{2}$ have no common eigenvalues over $\overline{\mathbb{F}}_{q}$. Let $t=\operatorname{diag}\left(s_{1}, s_{2}\right), s \in G^{*}$ be such that $\eta(s)=t$ (such $s$ exists as $\eta\left(G^{*}\right)=\Omega(V)$ ). Then $C_{\mathrm{O}(V)}(t)=C_{\mathrm{O}\left(V_{1}\right)}\left(s_{1}\right) \times C_{\mathrm{O}\left(V_{2}\right)}\left(s_{2}\right)$; it follows from Lemma 2.5 (3) that 
$C_{\mathrm{SO}(\mathrm{V})}(t)=C_{\mathrm{SO}\left(\mathrm{V}_{1}\right)}\left(s_{1}\right) \times C_{\mathrm{SO}\left(\mathrm{V}_{2}\right)}\left(s_{2}\right)$, and also that $C_{\mathrm{SO}(V)}(t), C_{\mathrm{SO}\left(V_{1}\right)}\left(s_{1}\right)$ and $C_{\mathrm{SO}\left(V_{2}\right)}\left(s_{2}\right)$ are finite reductive groups. So

$$
v\left(C_{\mathrm{SO}(V)}(t)\right)=v\left(C_{\mathrm{SO}\left(V_{1}\right)}\left(s_{1}\right)\right) \cdot v\left(C_{\mathrm{SO}\left(V_{2}\right)}\left(s_{2}\right)\right)=\beta(5) \beta(n-5) .
$$

By Lemma 2.10, v( $\left.C_{G^{*}}(s)\right)=\beta(5) \beta(n-5)$, so we are done if $n$ is even and $n>6$. If $n$ is odd, then $n-5 \equiv 0$ or $2 \bmod 4$; in both cases, $\beta(5) \beta(n-5)=\beta(n)$ by Lemma 2.1, provided $n-5 \geq 4$, whence the result.

(1) If $n$ is odd, this is already proven in Lemma 4.6. Suppose that $n$ is even. Suppose the contrary, and let $s \in G^{*}$ be such that $v\left(C_{G^{*}}(s)\right)>\beta(5) \beta(n-5)$.

Choose a decomposition

$$
V=\left(\bigoplus_{i=1}^{k} V_{i}\right) \oplus\left(\bigoplus_{j=1}^{l} V_{j}\right)
$$

described after Lemma 4.2; in particular, each term is a minimal non-degenerate $s$ stable subspace of $V$, each $V_{j}(j=1, \ldots, l)$ is minimal and each $V_{i}(i=1, \ldots, k)$ is the sum of two minimal $s$-stable subspaces of $V$. By Corollary 4.4,

$$
C_{G}(s) \cong \prod_{i=1}^{k} \mathrm{GL}_{d_{i}}\left(q^{l_{i}}\right) \times \prod_{j=1}^{l} \mathrm{U}_{e_{j}}\left(q^{m_{j}}\right),
$$

where $\operatorname{dim} V_{i}=2 d_{i} l_{i}, \operatorname{dim} V_{j}=2 e_{j} m_{j}$, so $\sum_{i} d_{i} l_{i}+\sum e_{j} m_{j}=n$. Note that each $V_{i}$ has Witt defect 0. By [8, Proposition 2.5.11], at least one $V_{j}$ has Witt defect 1 ; in particular, $l \neq 0$.

Observe first that the case $k=0$ and $l=1$ does not hold. Indeed, otherwise, $n=e_{j} m_{j}$ and $C_{G^{*}}(s) \cong \mathrm{U}_{e_{j}}\left(q^{m_{j}}\right)$. By Lemma 4.5, $v\left(C_{G^{*}}(s)\right) \leq p\left(n^{\prime}\right)$, where $n^{\prime}$ is the odd part of $n$. Then $p\left(n^{\prime}\right) \leq p\left(\frac{n}{2}\right)$ as $n$ is even, and $p\left(\frac{n}{2}\right) \leq \beta\left(\frac{n}{2}\right)$. By Lemma 2.2, we have $\beta\left(\frac{n}{2}\right)<7 \beta(n-5)$ and $\beta\left(\frac{n}{2}\right)<3 \beta(n-3)$. In the latter case, if $n-3 \equiv 1 \bmod 4$, then $3 \beta(n-3)=3 \cdot 7 \cdot 5^{(n-8) / 4}$, and this is less than

$$
\beta(5) \cdot \beta(n-5)=7 \cdot 77 \cdot 5^{(n-16) / 4}
$$

(as $n-5 \equiv 3 \bmod 4$ ). This is a contradiction.

Choose $j$ so that the Witt defect of $V_{j}$ is 1 . Set $W=V_{j}^{\perp}$, so $W \neq 0$ is the sum of all terms in the above decomposition but $V_{j}$. Then the Witt defect of $W$ equals 0 . Let $s_{j}, s^{\prime}$ be the restriction of $s$ to $V_{j}, W$, respectively. We show that $n_{j}:=e_{j} m_{j}$ is odd. Indeed, by Lemma 4.6, $v\left(C_{\mathrm{SO}(V)}(s)\right)=v\left(C_{\mathrm{SO}(W)}\left(s^{\prime}\right)\right) \cdot v\left(C_{\mathrm{SO}(W)}\left(s^{\prime}\right)\right)$, and $v\left(C_{\mathrm{SO}(W)}\left(s^{\prime}\right)\right) \leq \beta\left(n-n_{j}\right)$ by Lemma 4.7. If $n_{j}$ is even, then, by Lemma 4.5, 
$v\left(C_{\mathrm{SO}\left(V_{j}\right)}\left(s_{j}\right)\right)=p\left(n_{j}^{\prime}\right)$, where $n_{j}^{\prime}$ is the odd part of $n_{j}$. By Theorem 1.2, we have $p\left(n_{j}^{\prime}\right) \leq \beta\left(\frac{n_{j}}{2}\right)$. By Lemma $2.2, \beta\left(\frac{n_{j}}{2}\right) \leq 3 \beta\left(n_{j}-3\right)$. Then

$$
3 \beta\left(n_{j}-3\right) \cdot \beta\left(n-n_{j}\right) \leq 3 \beta(n-3)
$$

by Lemma 2.1. By the above, this is less than $\beta(5) \beta(n-5)$.

So $n_{j}$ must be odd, and hence $v\left(C_{G^{*}}(s)\right) \leq \beta^{\prime}(n)=\max _{a \text { odd }} \beta(a) \beta(n-a)$. If $n>6$, then $\beta^{\prime}(n)=\beta(5) \beta(n-5)$ by Lemma 2.3 , as required.

Recall that $\alpha(n), \alpha^{+}(n), \alpha^{-}(n)$ denote the number of unipotent characters of the group $\operatorname{Sp}_{2 n}(q), \operatorname{Spin}_{2 n}^{+}(q), \operatorname{Spin}_{2 n}^{-}(q)$, respectively, and note as well that $v\left(\operatorname{Spin}_{2 n+1}(q)\right)=\alpha(n)$.

An essential role in what follows is played by Lemmas 4.9 and 4.10 which generalize Lemma 3.5 to other classical groups.

Lemma 4.9. Let $G^{*} \in\left\{\operatorname{Spin}_{2 n}^{+}(q), \operatorname{Spin}_{2 n}^{-}(q), \operatorname{Sp}_{2 n}(q)\right\}, q$ even. Let $a, c \geq 0$ be integers such that $a+c=n$ and if $G^{*}=\operatorname{Spin}_{2 n}^{-}(q)$, then $c<n$. If $q \geq n-a+5$, then there exists a semisimple element $s \in G^{*}$ such that

$$
v\left(C_{G^{*}}(s)\right)= \begin{cases}\alpha(a) \beta(c) & \text { if } G^{*}=\operatorname{Sp}_{2 n}(q), \\ \alpha^{+}(a) \beta(c) & \text { if } G^{*}=\operatorname{Spin}_{2 n}^{+}(q), \\ \alpha^{-}(a) \beta(c) & \text { if } G^{*}=\operatorname{Spin}_{2 n}^{-}(q) .\end{cases}
$$

Proof. Let $V$ be the natural module for $G^{*}$. (Note that $\operatorname{Spin}(V) \cong \Omega(V)$.) Then $V$ contains a non-degenerate subspace $W$, say, of dimension $2 c$ and of Witt defect 0 . Set $H=\left\{g \in G^{*}: g x=x\right.$ for every $\left.x \in W^{\perp}\right\}$. Then $H \cong \operatorname{Spin}_{2 c}^{+}(q)$ or $\operatorname{Sp}_{2 c}(q)$. By Lemma 4.7 and its proof, there is an element $h \in H$ such that $v\left(C_{H}(h)\right)=\beta(c)$ and $h$ does not have eigenvalue 1 on $W$. Then

$$
C_{G^{*}}(h) \cong C_{H}(h) \times X, \quad \text { where } X \cong \operatorname{Sp}_{2 a}(q) \text { or } \operatorname{Spin}_{2 a}^{ \pm}(q) .
$$

By Lemma 4.6,

$$
v\left(C_{G}(h)\right)=v\left(C_{H}(h)\right) \cdot v(X)=\beta(c) \cdot x,
$$

where $x=\alpha^{+}(a), \alpha^{-}(a)$ or $\alpha(a)$ when $G^{*} \cong \operatorname{Spin}_{2 n}^{+}(q), \operatorname{Spin}_{2 n}^{-}(q), \operatorname{Sp}_{2 n}(q)$, respectively. This is recorded in the statement.

Lemma 4.10. Let $G^{*} \in\left\{\operatorname{Spin}_{2 n+1}(q), \operatorname{Spin}_{2 n}^{+}(q), \operatorname{Spin}_{2 n}^{-}(q), \operatorname{Sp}_{2 n}(q)\right\}, q$ odd, and let $a, b, c \geq 0$ be integers such that $a+b+c=n, a \neq 1, b \neq 1$, and if $G^{*}=\operatorname{Spin}_{2 n}^{-}(\bar{q})$, then $b+c<n$. If $G^{*} \neq \operatorname{Sp}_{2 n}(q)$, then suppose that $\frac{b(q-1)}{2}$ is 
even. Suppose that $q \geq n-a-b+5$. Then there exists a semisimple element $s \in G^{*}$ such that

$$
v\left(C_{G^{*}}(s)\right)= \begin{cases}\alpha(a) \alpha(b) \beta(c) & \text { if } G^{*}=\operatorname{Sp}_{2 n}(q), \\ \alpha(a) \alpha^{+}(b) \beta(c) & \text { if } G^{*}=\operatorname{Spin}_{2 n+1}(q), \\ \alpha^{+}(a) \alpha^{+}(b) \beta(c) & \text { if } G^{*}=\operatorname{Spin}_{2 n}^{+}(q), \\ \alpha^{-}(a) \alpha^{+}(b) \beta(c) & \text { if } G^{*}=\operatorname{Spin}_{2 n}^{-}(q) .\end{cases}
$$

Proof. Let $V$ be the natural module for $G^{*}$ and the respective classical group. Consider an orthogonal decomposition $V=W_{1} \oplus W_{2} \oplus W_{3}$, where $\operatorname{dim} W_{1}=2 a$ or $2 a+1, \operatorname{dim} W_{2}=2 b$ and $W_{3}=\left(W_{1}+W_{2}\right)^{\perp}$. If $V$ is orthogonal, choose both $W_{2}, W_{3}$ to be of Witt defect 0 . The condition $b+c<n$ makes this possible if $G^{*}=\operatorname{Spin}_{2 n}^{-}(q)$; in the other cases, this is well known to be possible.

Choose a basis

$$
B=\left\{b_{0}, b_{1}, \ldots, b_{2 n}\right\} \quad \text { in } V,
$$

where $b_{0}$ is dropped unless $G^{*}=\operatorname{Spin}_{2 n+1}(q)$. We can assume that

$$
b_{2 n-2 c+1}, \ldots, b_{2 n} \in W_{3}, \quad b_{2 n-2 c-2 b+1}, \ldots, b_{2 n-2 c} \in W_{2}
$$

and the remaining elements of $B$ are in $W_{1}$. With respect to this basis, consider the matrix $t=\operatorname{diag}\left(\mathrm{Id},-\operatorname{Id}_{2 b}, s^{\prime}\right)$, where $s^{\prime}$ is in $\Omega_{2 c}^{+}(q)$ or $\operatorname{Sp}_{2 c}(q)$. (Note that $-\mathrm{Id}_{2 b} \in \Omega\left(W_{2}\right)$ by Lemma 4.1.) By Lemma 4.7 and its proof, we can choose $s^{\prime}$ to be such that \pm 1 are not eigenvalues of $s^{\prime}$ and the number of unipotent characters of $C_{\mathrm{SO}\left(W_{3}\right)}\left(s^{\prime}\right)$ or $C_{\mathrm{Sp}\left(W_{3}\right)}\left(s^{\prime}\right)$ equals $\beta(c)$.

If $G^{*}=\operatorname{Sp}_{2 n}(q)$, then $C_{G^{*}}(t)=\operatorname{Sp}\left(W_{1}\right) \times \operatorname{Sp}\left(W_{2}\right) \times C_{\mathrm{Sp}\left(W_{3}\right)}\left(s^{\prime}\right)$, and the result follows as $v\left(\operatorname{Sp}\left(W_{1}\right)\right)=\alpha(a)$ and $v\left(\operatorname{Sp}\left(W_{2}\right)\right)=\alpha(b)$.

Suppose that $G^{*}$ is orthogonal. Then $v\left(\operatorname{SO}\left(W_{2}\right)\right)=\alpha^{+}(b)$ as $W_{2}$ is of Witt defect 0 , whereas $v\left(\operatorname{SO}\left(W_{1}\right)\right)=\alpha(a), \alpha^{+}(a)$ or $\alpha^{-}(a)$ depending on whether $G^{*}=\operatorname{Spin}_{2 n+1}(q), \operatorname{Spin}_{2 n}^{+}(q), \operatorname{Spin}_{2 n}^{-}(q)$, respectively. So again, the result follows from Lemma 2.10 .

\section{Some relations between $\alpha(n), \alpha^{+}(n), \alpha^{-}(n)$ and $\beta(n)$}

For $x \in \mathbb{R}$, let $[x]$ denote the maximum integer that does not exceed $x$.

The enumeration of unipotent characters in our context has a nice combinatorial description (see [11, Theorem 8.2] or [3, Section 13.8]); for computing $\alpha(n)$, $\alpha^{+}(n)$ and $\alpha^{-}(n)$ for small $n$ (Table 2), we use Lusztig's formulae [11, §3] expressing these functions in terms of $p(m)$ with $m \leq n$. 
Lemma 5.1. For $n \in \mathbb{N}$ odd, $\alpha^{-}(n)=\alpha^{+}(n)$, and for all $n \in \mathbb{N}$,

$$
\alpha^{-}(n) \leq \alpha^{+}(n) \leq \alpha(n) .
$$

Proof. From Lusztig's generating function [11, (3.4.2)], $\alpha^{+}(n)-\alpha^{-}(n)=0$ for $n$ odd, and $\alpha^{+}(n)-\alpha^{-}(n)=2 p\left(\frac{n}{2}\right)$ for $n$ even, so always $\alpha^{+}(n) \geq \alpha^{-}(n)$.

Let $p_{2}(n)$ denote the number of pairs of partitions that sum up to $n$; hence $p_{2}(n)=\sum_{m=0}^{n} p(m) p(n-m)$. Again from [11], we have

$$
\begin{aligned}
\alpha^{+}(n) & =\frac{1}{2} p_{2}(n)+\frac{3}{4}\left(1+(-1)^{n}\right) p\left(\frac{n}{2}\right)+\sum_{j>1 \text { even }} p_{2}\left(n-j^{2}\right), \\
\alpha(n) & =\sum_{j \geq 0} p_{2}\left(n-\left(j^{2}+j\right)\right) .
\end{aligned}
$$

For $n \leq 13$, the claim is easily checked directly (Table 2 ). For $n \geq 14$, the easy inequality $\frac{3}{2} p\left(\frac{n}{2}\right)<p(n-6)+p(n-7)<p_{2}(n-6)$ and a comparison of the summands in the sums above gives the claim.

Proposition 5.2. For $n \leq 43, \alpha(n)>\beta(n)$, and for all $n>43, \alpha(n)<\beta(n)$.

Proof. For $n \leq 43$, the stated inequalities for $\alpha(n)$ hold by computation (Table 2); these also show that $\alpha(n)<\beta(n)$ for $44 \leq n \leq 300$.

For $n>43$, we use very rough estimates to give an upper bound for $\alpha(n)$. First we have $p(n)<2^{[n / 2]+1}$ for all $n$ (for example, use [1]). Hence

$$
p_{2}(m)=\sum_{i=0}^{m} p(i) p(m-i) \leq(m+1) 2^{[m / 2]+2} \text { for all } m .
$$

Applying this, for any $n \geq 2$, we have

$$
\alpha(n)=\sum_{j \geq 0} p_{2}\left(n-\left(j^{2}+j\right)\right) \leq\left(n^{2}-1\right) 2^{[n / 2]+2} .
$$

One easily checks that $\alpha(n) \leq\left(n^{2}-1\right) 2^{[n / 2]+2}<5^{(n-3) / 4}$ for $n \geq 244$. Using Theorem 1.2, we conclude that $\alpha(n)<\beta(n)$ for all $n>43$.

Proposition 5.3. For $2<n \leq 38$, we have $\beta(n)<\alpha^{-}(n) \leq \alpha^{+}(n)$. For all $n \geq 39$, we have $\alpha^{-}(n) \leq \alpha^{+}(n)<\beta(n)$.

Proof. For $2<n \leq 43$, the stated inequalities hold by Table 2; for $n>43$, these follow from Lemma 5.1 and Proposition 5.2. 
Corollary 5.4. Let $n>43$. Then, for fixed $n$ but varying $a$, the maximum of each function $\alpha(a) \beta(n-a), \alpha^{+}(a) \beta(n-a)$ and $\alpha^{-}(a) \beta(n-a)$ is attained for $a \leq 43$.

Proof. Suppose on the contrary that the maximum is attained at some $a>43$. Then $\alpha^{-}(a) \beta(n-a) \leq \alpha^{+}(a) \beta(n-a) \leq \alpha(a) \beta(n-a)<\beta(a) \beta(n-a) \leq \beta(n)$. As $n>43$, by Theorem 1.2, we have

$$
\begin{aligned}
\beta(n)=5 \beta(n-4) & <10 \beta(n-4)=\alpha^{-}(4) \beta(n-4) \\
& <\alpha^{+}(4) \beta(n-4)<\alpha(4) \beta(n-4),
\end{aligned}
$$

a contradiction.

5.1 The products $\alpha(a) \beta(n-a), \alpha^{+}(a) \beta(n-a)$ and $\alpha^{-}(a) \beta(n-a)$

Lemma 5.5. The following statements hold.

(1) If $13<a \leq 43$, then

$$
\begin{aligned}
\alpha(a+4) & <5 \alpha(a), \\
\alpha^{+}(a+4) & <5 \alpha^{+}(a), \\
\alpha^{-}(a+4) & <5 \alpha^{-}(a) .
\end{aligned}
$$

(1a) If $0<a \leq 13$, then

$$
\begin{aligned}
\alpha(a+4) & >5 \alpha(a), \\
\alpha^{+}(a+4) & >5 \alpha^{+}(a), \\
\alpha^{-}(a+4) & >5 \alpha^{-}(a) .
\end{aligned}
$$

(2) If $13<a \leq 43$, then

$$
\begin{gathered}
\alpha(a+4) \beta(m)<\alpha(a) \beta(m+4), \\
\alpha^{+}(a+4) \beta(m)<\alpha(a)^{+} \beta(m+4), \\
\alpha^{-}(a+4) \beta(m)<\alpha^{-}(a) \beta(m+4)
\end{gathered}
$$

for every integer $m \geq 0$.

(3) If $a \leq 13, m>3, m \neq 5,6,11$, then

$$
\begin{gathered}
\alpha(a) \beta(m)<\alpha(a+4) \beta(m-4), \\
\alpha^{+}(a) \beta(m)<\alpha^{+}(a+4) \beta(m-4), \\
\alpha^{-}(a) \beta(m)<\alpha^{-}(a+4) \beta(m-4) .
\end{gathered}
$$

More precisely,

$$
\alpha(a) \beta(m)<\alpha(a+4) \beta(m-4)
$$

if $m=11, a<13$ or $m=6, a<12$ or $m=5, a<8$. 
(4) If $a<n$, then the maximum of

$$
\alpha(a) \beta(n-a), \quad \alpha^{+}(a) \beta(n-a) \quad \text { and } \quad \alpha^{-}(a) \beta(n-a)
$$

is attained for $a \leq 17$. If $n>24$, then, additionally, $a>13$.

Proof. (1) and (1a) follow directly by Table 2 .

(2) By Theorem 1.2 and Table 1 , we have $5 \beta(m) \leq \beta(m+4)$, so the claim follows from (1). (Note that $5 \beta(m)=\beta(m+4)$ for $m \neq 1,2,7$.)

(3) If $m>3, m \neq 5,6,11$, then $5 \beta(m-4)=\beta(m)$. Therefore, by (1a),

$$
\alpha(a+4) \beta(m-4)>5 \alpha(a) \beta(m-4)=\alpha(a) \beta(m),
$$

similarly for $\alpha^{+}(a), \alpha^{-}(a)$ in place of $\alpha(a)$.

Let $m=11$. Then $\beta(11)=77, \beta(7)=15$, so $\beta(11)=\frac{77}{15} \beta(7)$. So the result follows if $\alpha(a+4)>\frac{77}{15} \alpha(a)$. This is true if $a<13$.

Let $m=6$. Then $\beta(6)=11, \beta(2)=2$, so $\beta(6)=\frac{11}{2} \beta(2)$. So the result follows if $\alpha(a+4)>\frac{11}{2} \alpha(a)$. This is true for $a<12$.

Let $m=5$. Then $\beta(5)=7, \beta(1)=1$, so $\beta(5)=7 \beta(1)$. So the result follows if $\alpha(a+4)>7 \alpha(a)$. This is true for $a<8$.

(4) By Corollary 5.4, we may assume that $a \leq 43$. Suppose that $a>17$. Then, by (2), $\alpha(a) \beta(n-a)<\alpha(a-4) \beta(n-a+4)$, a contradiction. If $a \leq 13$, then $n>24$ implies $n-a>11$, so $\alpha(a) \beta(n-a)<\alpha(a+4) \beta(n-a-4)$ by (3), similarly for $\alpha^{+}(a), \alpha^{-}(a)$ in place of $\alpha(a)$.

Proposition 5.6. The following statements hold.

(1) For $n<18$, the maximum of $\alpha(a) \beta(n-a), \alpha^{+}(a) \beta(n-a), \alpha^{-}(a) \beta(n-a)$ is attained for $a=n$.

(2) Let $n \geq 18$. Then the maximum of $\alpha(a) \beta(n-a)$ is attained for $a=16,15,14$, 15 when $n \equiv 0,1,2,3 \bmod 4$.

(3) The maximum of $\alpha^{+}(a) \beta(n-a)$ and of $\alpha^{-}(a) \beta(n-a)$ is attained for $a=16$, $17,14,15$ when $n \equiv 0,1,2,3 \bmod 4$, respectively (in particular, $n \equiv a \bmod 4$ ).

Proof. By computer calculation, the claim is easily checked up to $n=29$. Let $n>29$. By Lemma 5.5 (4), the maximum of each of these functions is attained for $a$ with $13<a \leq 17$. Then $n-a>7$. Write $n-a=r+4 k$, where $7<r \leq 11$ and $k \geq 0$ is an integer. By Theorem 1.2, $\beta(n-a)=5^{k} \beta(r)$. So

$$
\alpha(a) \beta(n-a)=5^{k} \alpha(a) \beta(r), \quad \text { where } a+r<29 \text {. }
$$


By the above, the maximum of $\alpha(a) \beta(r)$ is attained for $a=16,15,14,15$ if $a+r$ is congruent to $0,1,2,3 \bmod 4$, respectively. Say, if $4 \mid(a+r)$, then $a=16$, and

$$
\alpha(a) \beta(n)=5^{k} \alpha(a) \beta(r) \leq 5^{k} \alpha(16) \beta(r)=\alpha(16) \beta(n),
$$

whence the result. The other cases are similar, as well as the cases with $\alpha^{+}(a)$, $\alpha^{-}(a)$ in place of $\alpha(a)$.

\subsection{The products $\alpha(a) \alpha(b) \beta(n-a-b)$ and $\alpha(a) \alpha^{+}(b) \beta(n-a-b)$}

Lemma 5.7. Let $n, a, b \geq 0$ be integers such that $a+b \leq n$. For $n$ fixed, the maximum of

$$
\begin{array}{ll}
\alpha(a) \alpha(b) \beta(n-a-b), & \alpha(a) \alpha^{+}(b) \beta(n-a-b), \\
\alpha^{+}(a) \alpha^{+}(b) \beta(n-a-b), & \alpha^{+}(a) \alpha^{-}(b) \beta(n-a-b)
\end{array}
$$

is attained for $a \leq 17, b \leq 17$.

Furthermore,

(1) if $n-a-b>11$, then $a>13, b>13$;

(2) if $n>45$, then $a>13, b>13$.

Proof. The first statement follows from Corollary 5.4 and Lemma 5.5 (4). Furthermore, $n-a>11$ and $n-b>11$. Suppose that $a \leq 13$. Then, by Lemma 5.5 (2), $\alpha(a) \alpha(b) \beta(n-a-b)<\alpha(a+4) \alpha(b) \beta(n-a-b-4)$, a contradiction, similarly for the other three functions, as well as for $b \leq 13$, whence (1). In addition, if $n>45$, then $n-a-b>11$ as $a+b \leq 34$, whence (2).

Proposition 5.8. Let $n, a, b \geq 0$ be integers, $n \geq a+b$.

In the table below, we record for each of the functions $\alpha(a) \alpha(b) \beta(n-a-b)$, $\alpha(a) \alpha^{+}(b) \beta(n-a-b), \alpha(a)^{+} \alpha^{+}(b) \beta(n-a-b)$ and $\alpha(a) \alpha^{-}(b) \beta(n-a-b)$ the pairs $(a, b)$ where the functions attain their maximum for $n \geq 28$ or $n \geq 29$; for the first and third function, we list the pairs with $a \geq b$. (Here we write $\equiv_{4}$ for the congruence modulo 4.)

\begin{tabular}{llllll}
\hline function & bound & $n \equiv_{4} 0$ & $n \equiv_{4} 1$ & $n \equiv_{4} 2$ & $n \equiv_{4} 3$ \\
\hline$\alpha(a) \alpha(b) \beta(n-a-b)$ & $n \geq 28$ & $(14,14)$ & $(15,14)$ & $(15,15)$ & $(16,15)$ \\
$\alpha(a) \alpha^{+}(b) \beta(n-a-b)$ & $n \geq 29$ & $(16,16)$ & $(15,14)$ & $(14,16)$ & $(15,16)$ \\
$\alpha^{+}(a) \alpha^{+}(b) \beta(n-a-b)$ & $n \geq 29$ & $(16,16)$ & $(15,14)$ & $(16,14)$ & $(16,15)$ \\
$\alpha^{+}(a) \alpha^{-}(b) \beta(n-a-b)$ & $n \geq 29$ & $(16,16)$ & $(14,15)$ & $(14,16)$ & $(16,15)$ \\
\hline
\end{tabular}


Proof. The assertion was checked to hold for $n \leq 50$ by computer, so we may assume $n>50$. Lemma 5.7 shows that the values $a, b$ at which all four products in question attain their maximum satisfy $13<a, b<18$. Write $n=4 k+r$, with $a+b+7 \leq r \leq a+b+10$, and some integer $k \geq 0$. Then $r \leq 44$. Let $\gamma_{n}(a, b)$ stand for any of the functions above. As $r>7$, by Theorem 1.2, we have $\beta(n-a-b)=\beta(r-a-b) \beta(4)^{k}$, and hence $\gamma_{n}(a, b)=\beta(4)^{k} \gamma_{r}(a, b)$. Since $35 \leq r \leq 44$, the claim holds for $r$, and the result follows.

Remarks. (1) For all $n<32$, the maximum of $\alpha(a) \alpha(b) \beta(n-a-b)$ is attained for pairs $(a, b)$ such that $a+b=n$.

(2) For $n \leq 33$, the maxima of the functions $\gamma_{n}(a, b)$ defined in the proof of Proposition 5.8 have been calculated by computer and are shown in Tables 3 and 4 at the end of the paper.

\section{Proof of the main results for $q$ even}

In this section, $q$ is even and $G^{*} \in\left\{\operatorname{Sp}_{2 n}(q)\right.$, $\left.\operatorname{Spin}_{2 n}^{ \pm}(q) \cong \Omega_{2 n}^{ \pm}(q)\right\}$. For $q$ large enough, we determine the maximum of $v\left(C_{G^{*}}(s)\right)$ when $s$ runs over the semisimple elements of $G^{*}$.

Let $V$ be a vector space of dimension $2 n$ over $\mathbb{F}_{q}$ viewed as the natural module for $G^{*}$, so $V$ is endowed with a suitable form defining $G^{*}$. Denote by $V_{1}$ the 1-eigenspace of $s$ on $V$. By Lemma 4.2, $V_{1}$ is non-degenerate and $\operatorname{dim} V_{1}=2 a$ is even. Set $W=V_{1}^{\perp}$, so $V=V_{1} \oplus W$. Let $s^{\prime}$ denote the restriction of $s$ to $W$. We keep this notation until the end of this section.

Lemma 6.1. Proposition 1.4 is true for q even.

Proof. Suppose that $G^{*} \cong \operatorname{Spin}_{2 n}^{ \pm}(q)$ (the proof for $G^{*}=\operatorname{Sp}_{2 n}(q)$ is similar and hence omitted). By Lemma 2.10,

$$
v\left(C_{G^{*}}(s)\right)=v\left(\mathrm{SO}\left(V_{1}\right)\right) \cdot v\left(C_{\mathrm{SO}(W)}\left(s^{\prime}\right)\right)=\alpha^{ \pm}(a) \cdot v\left(C_{\mathrm{SO}(W)}\left(s^{\prime}\right)\right) .
$$

By Lemma 4.6(1), v $\left(C_{\mathrm{SO}(W)}\left(s^{\prime}\right)\right) \leq \beta(n-a)$. If $n<18$, then the maximum of $\alpha^{ \pm}(a) \beta(n-a)$ is attained for $a=n$ (Proposition 5.6(1)), which is realized for $s=1$.

\subsection{Symplectic groups in even characteristic}

Let $G^{*}=\operatorname{Sp}_{2 n}(q)$. Then $C_{G^{*}}(s) \cong \operatorname{Sp}_{2 a}(q) \times C_{S p_{2(n-a)}(q)}\left(s^{\prime}\right)$, so

$$
v\left(C_{G^{*}}(s)\right)=\alpha(a) \cdot v\left(C_{S p_{2(n-a)}(q)}\left(s^{\prime}\right)\right),
$$


and we are to determine the maximum of this product. Recall that, by Lemma 4.6, $v\left(C_{S p_{2(n-a)}(q)}\left(s^{\prime}\right)\right) \leq \beta(n-a)$ as $s^{\prime}$ does not have eigenvalue 1.

Theorem 6.2. Theorem 1.5 is true for $G^{*}=\operatorname{Sp}_{2 n}(q)$, q even.

Proof. If $n$ is fixed and $a$ varies, the maximum of $\alpha(a) \beta(n-a)$ is attained for $a=16,15,14,15$ for $n \equiv 0,1,2,3 \bmod 4$, respectively; see Proposition 5.6. So $f(n)$ is a bound for the products $\alpha(a) \beta(n-a)$.

The values of $\alpha(a)$ for $a \leq 17$ are provided by Table 2 , and $\beta(n-a)$ is given by Table 1 . Using this, the result follows by easy computations. (For instance, $n \equiv 1 \bmod 4 \operatorname{implies} n-15 \equiv 2 \bmod 4$, and then $\beta(n-15)=11 \cdot 5^{n-21}$ by Table 1.)

Now we turn to the additional statement on the bound being attained for large $q$. By Lemma 4.9, if $n-a \leq q-5$, then there exists a semisimple element $s \in G^{*}$ such that

$$
v\left(C_{G^{*}}(s)\right)=\alpha(a) \beta(n-a) .
$$

This holds if $n-14 \leq q-5$, that is, $q \geq n-9$.

\subsection{Orthogonal groups in even characteristic}

In this case, we are to consider the groups $G^{*}=\operatorname{Spin}_{2 n}^{ \pm}(q) \cong \operatorname{SO}_{2 n}^{ \pm}(q)$. By Lemma 2.10, v $\left(C_{G^{*}}(s)\right)=v\left(\mathrm{SO}\left(V_{1}\right)\right) \cdot v\left(C_{\mathrm{SO}(W)}\left(s^{\prime}\right)\right)$, and $v\left(C_{\mathrm{SO}(W)}\left(s^{\prime}\right)\right) \leq \beta(n-a)$ by Lemma 4.6. So $\left|E_{S}\right|=v\left(C_{G^{*}}(s)\right) \leq \alpha^{ \pm}(a) \beta(n-a)$, where one chooses + if and only if $V_{1}$ is of Witt defect 0 .

Theorem 6.3. Theorem 1.5 is true if $G^{*}=\operatorname{Spin}_{2 n}^{ \pm}(q)$, q even. More precisely,

$$
\left|E_{S}\right|=v\left(C_{G^{*}}(s)\right) \leq \alpha^{ \pm}(a) \beta(n-a),
$$

where $a=16,17,14,15$ when $n \equiv 0,1,2,3 \bmod 4$, respectively, and $\left|\varepsilon_{S}\right| \leq f^{ \pm}(n)$, where $f^{ \pm}(n)$ are as in Theorem 1.5.

Proof. Let $x, y$ denote the Witt defect of $V_{1}, W$, respectively.

(i) $(x, y)=(0,0)$. Then $G^{*}=\operatorname{Spin}_{2 n}^{+}(q)$ and $v\left(C_{G^{*}}(s)\right) \leq \alpha^{+}(a) \beta(n-a)$.

(ii) $(x, y)=(1,1)$. Then $G^{*}=\operatorname{Spin}_{2 n}^{+}(q)$ and $v\left(C_{G^{*}}(s)\right) \leq \alpha^{-}(a) \beta(n-a)$.

(iii) $(x, y)=(1,0)$. Then $G^{*}=\operatorname{Spin}_{2 n}^{-}(q)$ and $v\left(C_{G^{*}}(s)\right) \leq \alpha^{-}(a) \beta(n-a)$.

(iv) $(x, y)=(0,1)$. Then $G^{*}=\operatorname{Spin}_{2 n}^{-}(q)$ and $v\left(C_{G^{*}}(s)\right) \leq \alpha^{+}(a) \beta(n-a)$ if $n-a$ is odd, and $v\left(C_{G^{*}}(s)\right) \leq \alpha^{+}(a) \beta^{\prime}(n-a)$ if $n-a$ is even; see Lemma 4.8 (and Lemma 2.3 for the values of $\beta^{\prime}$ ). 
Assume that $s$ is chosen so that $v\left(C_{G^{*}}(s)\right)$ is maximal. Then we show that cases (ii) and (iv) can be ignored for our purpose.

In case (ii), $\alpha^{-}(a) \leq \alpha^{+}(a)$, and by Lemma 4.9, $v\left(C_{G^{*}}(s)\right)=\alpha^{+}(a) \beta(n-a)$ for some $s \in G^{*}$. So we do not need to care whether the same maximum can be attained in case (ii). Then, using Proposition 5.6, we obtain the data for $f^{+}(n)$. For instance, if $n \equiv 1 \bmod 4$, then $a=17$ and $\alpha^{+}(17) \beta(n-17)=6007 \cdot 5^{(n-17) / 4}$.

Suppose, on the contrary, that (iv) holds. We first obtain an upper bound for $\max _{a: n-a \text { even }} \alpha^{+}(a) \beta^{\prime}(n-a)$ and $\max _{a: n-a \text { odd }} \alpha^{+}(a) \beta(n-a)$, and next show that these are less than $\max _{a} \alpha^{-}(a) \beta(n-a)$, which will yield the stated claim.

Assume first that $n-a$ is even. By Lemma 2.3, we have

$$
\beta^{\prime}(n-a)= \begin{cases}539 \cdot \beta(n-a-16) & \text { if } n-a \equiv 0 \bmod 4, n-a \geq 16 \\ 49 \cdot \beta(n-a-10) & \text { if } n-a \equiv 2 \bmod 4, n-a>6\end{cases}
$$

So $\alpha^{+}(a) \beta^{\prime}(n-a)=539 \cdot \alpha^{+}(a) \beta(n-a-16)$ and $49 \cdot \alpha^{+}(a) \beta(n-a-10)$ accordingly; by Proposition 5.6, if $n-16 \geq 18$, respectively, $n-10 \geq 18$, then the maximum of these is attained when $a=16$ if $n \equiv n-16 \equiv 0 \bmod 4$, respectively, when $a=14$ if $n-10 \equiv 0 \bmod 4$, equivalently, $n \equiv 2 \bmod 4$. Thus, if $n-a$ is even, then $\alpha^{+}(a) \beta^{\prime}(n-a)$ does not exceed

$$
\left\{\begin{aligned}
539 \alpha^{+}(16) \beta(n-32)=539 \cdot 4110 \cdot 5^{(n-32) / 4} & \text { if } n-a \equiv 0, n \equiv 0 \bmod 4, \\
539 \alpha^{+}(17) \beta(n-33)=539 \cdot 6007 \cdot 5^{(n-33) / 4} & \text { if } n-a \equiv 0, n \equiv 1 \bmod 4, \\
539 \alpha^{+}(14) \beta(n-30)=539 \cdot 1836 \cdot 5^{(n-30) / 4} & \text { if } n-a \equiv 0, n \equiv 2 \bmod 4, \\
539 \alpha^{+}(15) \beta(n-31)=539 \cdot 2730 \cdot 5^{(n-31) / 4} & \text { if } n-a \equiv 0, n \equiv 3 \bmod 4, \\
49 \alpha^{+}(16) \beta(n-26)=539 \cdot 4110 \cdot 5^{(n-32) / 4} & \text { if } n-a \equiv 2, n \equiv 0 \bmod 4, \\
49 \alpha^{+}(17) \beta(n-27)=539 \cdot 6007 \cdot 5^{(n-33) / 4} & \text { if } n-a \equiv 2, n \equiv 1 \bmod 4, \\
49 \alpha^{+}(14) \beta(n-24)=539 \cdot 1836 \cdot 5^{(n-30) / 4} & \text { if } n-a \equiv 2, n \equiv 2 \bmod 4, \\
49 \alpha^{+}(15) \beta(n-25)=539 \cdot 2730 \cdot 5^{(n-31) / 4} & \text { if } n-a \equiv 2, n \equiv 3 \bmod 4 .
\end{aligned}\right.
$$

Indeed, here $n-i \equiv 0 \bmod 4$ for $i=32,33,30,31$ in the last four rows, so

$$
\beta(n-i+6)=\beta(6) \beta(n-i)=11 \beta(n-i)
$$

for these $i$ by Theorem 1.2 , whence the equalities there as $49 \cdot 11=539$. (Note that we do not assert that these bounds are attained.)

Next we assume $n-a$ to be odd in case (iv). Then $\beta(n-a)=7 \beta(n-5-a)$ if $n-a \equiv 1 \bmod 4$, and $\beta(n-a)=77 \beta(n-11-a)$ if $n-a \equiv 3 \bmod 4$; see Theorem 1.2. By Proposition 5.6, applied to $\alpha^{+}(a) \beta(n-a-5), \alpha^{+}(a) \beta(n-a-11)$, 
if $n-11 \geq 18$, then $\alpha^{+}(a) \beta(n-a)$ with $n-a$ odd does not exceed the following values:

$$
\left\{\begin{array}{clrl}
7 \alpha^{+}(16) \beta(n-21) & =7 \cdot 4110 \cdot 5^{(n-21) / 4} & & \text { if } n-a \equiv 1, n \equiv 1 \bmod 4, \\
7 \alpha^{+}(17) \beta(n-22) & =7 \cdot 6007 \cdot 5^{(n-22) / 4} & & \text { if } n-a \equiv 1, n \equiv 2 \bmod 4, \\
7 \alpha^{+}(14) \beta(n-19) & =7 \cdot 1836 \cdot 5^{(n-19) / 4} & & \text { if } n-a \equiv 1, n \equiv 3 \bmod 4, \\
7 \alpha^{+}(15) \beta(n-20) & =7 \cdot 2730 \cdot 5^{(n-20) / 4} & & \text { if } n-a \equiv 1, n \equiv 0 \bmod 4, \\
77 \alpha^{+}(16) \beta(n-27) & =77 \cdot 4110 \cdot 5^{(n-27) / 4} & & \text { if } n-a \equiv 3, n \equiv 3 \bmod 4, \\
77 \alpha^{+}(17) \beta(n-28) & =77 \cdot 6007 \cdot 5^{(n-28) / 4} & & \text { if } n-a \equiv 3, n \equiv 0 \bmod 4, \\
77 \alpha^{+}(14) \beta(n-25) & =77 \cdot 1836 \cdot 5^{(n-25) / 4} & & \text { if } n-a \equiv 3, n \equiv 1 \bmod 4, \\
77 \alpha^{+}(15) \beta(n-26) & =77 \cdot 2730 \cdot 5^{(n-26) / 4} & & \text { if } n-a \equiv 3, n \equiv 2 \bmod 4 .
\end{array}\right.
$$

We have to compare this with $\max _{a} \alpha^{-}(a) \beta(n-a)$. By Proposition 5.6, this is equal to

$$
\begin{cases}\alpha^{-}(17) \beta(n-17)=6007 \cdot 5^{(n-17) / 4}=6007 \cdot 25 \cdot 5^{(n-25) / 4} & \text { if } n \equiv 1 \bmod 4, \\ \alpha^{-}(15) \beta(n-15)=2730 \cdot 5^{(n-15) / 4}=2730 \cdot 5^{3} \cdot 5^{(n-27) / 4} & \text { if } n \equiv 3 \bmod 4, \\ \alpha^{-}(16) \beta(n-16)=4066 \cdot 5^{(n-16) / 4}=4066 \cdot 5^{3} \cdot 5^{(n-28) / 4} & \text { if } n \equiv 0 \bmod 4, \\ \alpha^{-}(14) \beta(n-14)=1806 \cdot 5^{(n-14) / 4}=1806 \cdot 5^{3} \cdot 5^{(n-26) / 4} & \text { if } n \equiv 2 \bmod 4 .\end{cases}
$$

Then we conclude that the latter are greater than the former. So, in case (iv) with $n \geq 34$, the maximum of $\nu\left(C_{G^{*}}(s)\right)$ does not exceed $\alpha^{-}(a) \beta(n-a)$. The same trivially holds in case (iii). So the values for $f^{ \pm}(n)$ for $n \geq 34$ follow from the above.

For $n<34$, we use computer calculations.

Finally, we show that the bound $\left|\mathcal{E}_{s}\right| \leq f^{ \pm}(n)$ is attained for $q$ as stated. We have $13<a \leq 17$, so $n-a \leq n-14$ above. By Lemma 4.9, if $n-a \leq q-5$, then there exists a semisimple element $s \in G^{*}$ such that

$$
v\left(C_{G^{*}}(s)\right)=\alpha^{ \pm}(a) \beta(n-a) .
$$

This holds if $n-14 \leq q-5$, that is, $q \geq n-9$. So the result follows.

\section{Proof of the main results for $q$ odd}

In this section, we assume that $q$ is odd. Let $V$ be the natural $\mathbb{F}_{q} G^{*}$-module and $s \in G^{*}$ a semisimple element. Let $V_{1}$ and $V_{2}$ denote the 1 - and -1-eigenspaces of $s$ on $V$, respectively. These spaces are non-degenerate (if non-zero) and have even dimensions, except for the case where $\operatorname{dim} V$ is odd (Lemma 4.2). Set $\operatorname{dim} V_{1}=2 a$ or $2 a+1 \quad$ and $\quad \operatorname{dim} V_{2}=2 b, \quad$ where $0 \leq a, b \leq n$. 
Set $W=\left(V_{1}+V_{2}\right)^{\perp}$. Then $V=V_{1} \oplus V_{2} \oplus W$. One easily observes that $C_{G^{*}}(s)$ stabilizes $V_{1}, V_{2}$ and $W$. Let $s^{\prime}$ be the restriction of $s$ to $W$. As above, we have $\left|\mathcal{E}_{s}\right|=v\left(C_{G^{*}}(s)\right)$.

Lemma 7.1. Proposition 1.4 is true for $q$ odd.

Proof. Tables 3 and 4 at the end of the paper are obtained by computer calculations and give us the maximum of the functions in question. So we have to show that these coincide with the maximum of $\left|\mathcal{E}_{S}\right|$ in each case.

From a look at the tables, one observes that $n \leq 32$ implies $n=a+b$ (in the notation of the tables). Let $V$ be the natural module for $G^{*}$. Write $V=V_{1}+V_{2}$, where $V_{1}, V_{2}$ are non-degenerate subspaces of $V$ such that $\operatorname{dim} V_{1}=2 a$ or $2 a+1$, $\operatorname{dim} V_{2}=2 b$ and $V_{2}$ is of Witt defect 0 . This is always possible unless

$$
G^{*}=\operatorname{Spin}_{2 n}^{-}(q) \text { and } a=0 ;
$$

this happens only for $n=2,4,6$ (Table 4), but these cases are excluded in the statement. Consider the matrix $t=\operatorname{diag}\left(\operatorname{Id}_{2 a},-\mathrm{Id}_{2 b}\right) \in \mathrm{SO}(V)$ such that $V_{1}, V_{2}$ are the 1- and -1-eigenspace of $t$ on $V$. If $b=0$, then we set $t=\operatorname{Id}_{2 a}$ or $\operatorname{Id}_{2 a+1}$. From another look at the tables, we conclude that $b$ is always even, and hence $-\mathrm{Id} \in \Omega_{2 b}^{+}(q)$ for $b>0$ by Lemma 4.1. Then we take $s$ from the preimage of $t$ in $G^{*}$.

Remark. In case of $\mathrm{SO}_{2 n}^{-}(q)$ and $n=2,4,6$, one easily checks that the maximum of $\left|\mathcal{E}_{S}\right|$ is attained for $\alpha^{-}(2) \alpha^{+}(0)=2, \alpha^{-}(4) \alpha^{+}(0)=10$ and $\alpha^{-}(4) \alpha^{+}(2)=40$ respectively. If $G^{*}=\operatorname{Sp}_{64}(q)$, then $\max _{s \in G^{*}}\left|\mathcal{E}_{s}\right|=5 \alpha(14)^{2}$ by Theorem 7.2.

\section{1 $G^{*}$ is symplectic}

Theorem 7.2. Theorem 1.5 is true if $G^{*} \cong \operatorname{Sp}_{2 n}(q)$, q odd. More precisely,

$$
\left|\mathcal{E}_{S}\right|=v\left(C_{G^{*}}(s)\right) \leq \tau(n)=\max _{a+b \leq n} \alpha(a) \alpha(b) \beta(n-a-b),
$$

where $\tau(n)$ is as in Theorem 1.5.

Proof. One easily observes that $C_{G^{*}}(s)$ stabilizes $V_{1}, V_{2}$ and $W$. So

$$
C_{G^{*}}(s) \subset \operatorname{Sp}_{2 a}(q) \times \operatorname{Sp}_{2 b}(q) \times \operatorname{Sp}_{2(n-a-b)}(q),
$$

and in fact, $C_{G^{*}}(s)=\operatorname{Sp}_{2 a}(q) \times \operatorname{Sp}_{2 b}(q) \times C_{\mathrm{Sp}_{2(n-a-b)}(q)}\left(s^{\prime}\right)$. Therefore,

$$
v\left(C_{G^{*}}(s)\right)=\alpha(a) \alpha(b) \cdot v\left(C_{\mathrm{Sp}(W)}\left(s^{\prime}\right)\right) .
$$


By Lemma 4.6, $v\left(C_{\mathrm{Sp}(W)}\left(s^{\prime}\right)\right) \leq \beta(n-a-b)$. Therefore, if $s$ varies, $\left|\mathcal{E}_{S}\right|$ does not exceed the maximum of $\alpha(a) \alpha(b) \beta(n-a-b)$, where $a, b \geq 0$ and $a+b \leq n$. The values of $a, b$ for which the function $\alpha(a) \alpha(b) \beta(n-a-b)$ attains its maximum are determined in Table 3 for $n \leq 33$ and in Proposition 5.8 for $n \geq 28$. This yields the explicit expressions for $\tau(n)$ in Theorem 1.5.

It remains to show the additional statement in Theorem 1.5. By Lemma 4.10 (or Lemma 4.7 applied to $\left.\operatorname{Sp}_{2(n-a-b)}(q)\right)$, if $q \geq n-a-b+5$ with $a, b$ as above, there is a semisimple element $s \in G^{*}$ such that

$$
\left|\mathcal{E}_{S}\right|=v\left(C_{G^{*}}(s)\right)=\alpha(a) \alpha(b) \beta(n-a-b) .
$$

So the bound is attained, whence the result.

\section{2 $G^{*}=\operatorname{Spin}_{2 n+1}(q)$}

Theorem 7.3. Theorem 1.5 is true for $G^{*}=\operatorname{Spin}_{2 n+1}(q)$, $q$ odd. More precisely, $\left|\mathcal{E}_{S}\right|=v\left(C_{G^{*}}(s)\right) \leq \theta(n)=\max _{a+b \leq n} \alpha(a) \alpha(b) \beta(n-a-b)$, where $\theta(n)$ is as in Theorem 1.5 (4).

Proof. Suppose for a moment that $s$ is an arbitrary semisimple element in $G^{*}$. Then $\left|\mathcal{E}_{S}\right|=v\left(\mathrm{SO}\left(V_{1}\right)\right) \cdot v\left(\mathrm{SO}\left(V_{2}\right)\right) \cdot v\left(C_{\mathrm{SO}(W)}\left(s^{\prime}\right)\right)=\alpha(a) \alpha^{ \pm}(b) v\left(C_{\mathrm{SO}(W)}\left(s^{\prime}\right)\right)$ by Lemma 2.10 , where one chooses the sign + if and only if the Witt defect of $W$ is 1 . Therefore, we have $\left|\mathcal{E}_{s}\right| \leq \max _{a, b} \alpha(a) \alpha^{+}(b) \beta(n-a-b)$. By Proposition 5.8, if $n \geq 32$, then the maximum of $\alpha(a) \alpha^{+}(b) \beta(n-a-b)$ is attained for $(a, b)$ given in the table there (in particular, with $b$ even). The data $\alpha(i) \alpha^{+}(j)$ for $14 \leq i, j \leq 16$ follows from Table 3 . So the inequality $\left|\mathscr{E}_{S}\right| \leq \theta(n)$ follows.

For the additional statement in Theorem 1.5, we note that the existence of $s$ such that $\left|\varepsilon_{s}\right|=\theta(n)$ follows from Lemma 4.10 (provided $b$ is even, which is the case here).

\subsection{Orthogonal groups of even dimension}

In this subsection, we assume that $q$ is odd and $G^{*}=\operatorname{Spin}_{2 n}^{ \pm}(q)$.

By Lemma 2.10, $\left|\mathcal{E}_{S}\right|=v\left(C_{G^{*}}(s)\right)=v\left(\mathrm{SO}\left(V_{1}\right)\right) \cdot v\left(\mathrm{SO}\left(V_{2}\right)\right) \cdot v\left(C_{\mathrm{SO}(W)}\left(s^{\prime}\right)\right)$. As $\alpha^{-}(a) \leq \alpha^{+}(a)$ and $v\left(C_{\mathrm{SO}(W)}\left(s^{\prime}\right)\right) \leq \beta(n-a-b)$ (Lemma 4.6(1)), it follows that $\left|\mathcal{E}_{s}\right| \leq \max _{a, b} \alpha^{+}(a) \alpha^{+}(b) \beta(n-a-b)$. In turn, by Proposition 5.8, if $n \geq 32$, then the maximum of $\alpha^{+}(a) \alpha^{+}(b) \beta(n-a-b)$ is attained for

$$
(a, b)=(16,16),(15,14),(16,14),(15,16) \text { for } n \equiv 0,1,2,3 \bmod 4,
$$

respectively. In particular, $b \in\{14,16\}$ is even and $n-a-b \equiv 0 \bmod 4$. 
Theorem 7.4. Theorem 1.5 is true for $G^{*}=\operatorname{Spin}_{2 n}^{+}(q)$, $q$ odd. More precisely,

$$
\left|\mathcal{E}_{S}\right|=v\left(C_{G^{*}}(s)\right) \leq \theta^{+}(n)=\max _{a+b \leq n} \alpha^{+}(a) \alpha^{+}(b) \beta(n-a-b),
$$

where $\theta^{+}(n)$ is as in Theorem $1.5(5)$.

Proof. The comments prior to the theorem show that $\left|\mathcal{E}_{s}\right| \leq \theta^{+}(n)$, so we are left to show that the equality holds for some semisimple element $s \in G^{*}$. This follows from Lemma 4.10 as now $b \in\{14,16\}$ and $\frac{b(q-1)}{2}$ are even, so Lemma 4.10 applies.

Theorem 7.5. Theorem 1.5 is true for $s \in G^{*}=\operatorname{Spin}_{2 n}^{-}(q)$, $q$ odd.

Proof. Let $V$ be the natural module for $G^{*}$ and $s \in G^{*}$ an arbitrary semisimple element. As above, consider a decomposition $V=V_{1} \oplus V_{2} \oplus W$, where $V_{1}$ and $V_{2}$ are the 1- and -1-eigenspaces of $s$ on $V$ and $W=\left(V_{1}+V_{2}\right)^{\perp}$. By Lemma 2.10, $\left|\mathscr{E}_{s}\right|=v\left(C_{G^{*}}(s)\right)=v\left(\mathrm{SO}\left(V_{1}\right)\right) \cdot v\left(\mathrm{SO}\left(V_{2}\right)\right) \cdot v\left(C_{\mathrm{SO}(W)}\left(s^{\prime}\right)\right)$, where $s^{\prime}$ is the restriction of $s$ to $W$. As the Witt defect of $V^{*}$ equals 1 , for the Witt defects of $V_{1}, V_{2}$ and $W$, we have the following options:

(i) the Witt defect of $V_{1}$ is 1 , the two other are 0 ;

(ii) the Witt defect of $V_{2}$ is 1 , the two other are 0 ;

(iii) the Witt defect of $W$ is 1 , the two other are 0 ;

(iv) $V_{1}, V_{2}, W$ are of Witt defect 1 .

Suppose that $s$ is chosen so that $\left|\mathscr{E}_{s}\right|$ is maximal. Then (iv) can be ignored. Indeed, in this case, we have $v\left(C_{G^{*}}(s)\right)=\alpha^{-}(a) \alpha^{-}(b) \cdot v\left(C_{\mathrm{SO}(W)}\left(s^{\prime}\right)\right)$, where $v\left(C_{\mathrm{SO}(W)}\left(s^{\prime}\right)\right) \leq \beta(n-a-b)$ by Lemma 4.6. One can choose another element $s_{1} \in G^{*}$ for which the -1-eigenspace is the same as for $s$, the 1-eigenspace $U$, say, is of dimension $2 a$ and of Witt defect 0 , and for $W_{1}=\left(U+V_{2}\right)^{\perp}$, choose $s_{1}^{\prime} \in \Omega\left(W_{1}\right)$ so that $v\left(C_{\mathrm{SO}(W)}\left(s_{1}^{\prime}\right)\right)=\beta(n-a-b)$. This is possible as the Witt defect of $W_{1}$ is 0 , so $\Omega\left(W_{1}\right) \cong \Omega_{2(n-a-b)}^{+}(q)$; see Lemma 4.7. Then

$$
\begin{aligned}
\left|\mathcal{E}_{S_{1}}\right| & =v\left(C_{G^{*}}\left(s_{1}\right)\right)=v\left(\mathrm{SO}\left(V_{1}\right)\right) \cdot v\left(\mathrm{SO}\left(V_{2}\right)\right) \cdot v\left(C_{\mathrm{SO}(W)}\left(s_{1}^{\prime}\right)\right) \\
& =\alpha^{+}(a) \alpha^{-}(b) \cdot v\left(C_{\mathrm{SO}(W)}\left(s_{1}^{\prime}\right)\right)=\alpha^{+}(a) \alpha^{-}(b) \beta(n-a-b) .
\end{aligned}
$$

As $\alpha^{-}(a) \leq \alpha^{+}(a)$, we have $\left|\mathcal{E}_{S}\right| \leq\left|\mathcal{E}_{S_{1}}\right|$, so we can assume that (iv) does not hold.

Suppose first that $W$ is of Witt defect 0 . Then (i) or (ii) holds, and

$$
v\left(C_{G^{*}}(s)\right)= \begin{cases}\alpha^{-}(a) \alpha^{+}(b) v\left(C_{\mathrm{SO}(W)}\left(s^{\prime}\right)\right) & \text { in case (i), } \\ \alpha^{+}(a) \alpha^{-}(b) v\left(C_{\mathrm{SO}(W)}\left(s^{\prime}\right)\right) & \text { in case (ii). }\end{cases}
$$


By Lemma 4.6(1), $v\left(C_{\mathrm{SO}(W)}\left(s^{\prime}\right)\right) \leq \beta(n-a-b)$. By Proposition 5.8, the maximum of the function $\alpha^{-}(a) \alpha^{+}(b) \beta(n-a-b)$ is attained for

$$
(a, b)=(16,16),(15,14),(16,14),(15,16) \quad \text { for } n \equiv 0,1,2,3 \bmod 4,
$$

respectively, and the maximum of $\alpha^{+}(a) \alpha^{-}(b) \beta(n-a-b)$ is attained for

$$
(a, b)=(16,16),(14,15),(14,16),(16,15) \quad \text { for } n \equiv 0,1,2,3 \bmod 4 .
$$

By Lemma 4.6 (3) and Lemma 4.7, if $q>n-a-b+5$, then there is $s^{\prime}$ such that $v\left(C_{\mathrm{SO}(W)}\left(s^{\prime}\right)\right)=\beta(n-a-b)$; for the above values of $a, b$, it suffices to assume $q>n-27$.

In case (i), $b \in\{14,16\}$ is even and the Witt defect of $V_{2}$ is 0 , so $-\operatorname{Id}_{2 b} \in \Omega\left(V_{2}\right)$ by Lemma 4.1. It follows that $t \in \Omega_{2 n}^{-}(q)$, where $t=\operatorname{diag}\left(\operatorname{Id}_{2 a},-\operatorname{Id}_{2 b}, s^{\prime}\right)$ and $s^{\prime} \in \Omega(W)$ is such that \pm 1 are not eigenvalues of $s$ and

$$
v\left(C_{\mathrm{SO}(W)}\left(s_{1}^{\prime}\right)\right)=\beta(n-a-b) .
$$

Let $s \in G^{*}$ be such that $t$ is the matrix of $s$ on $V$. Then

$$
\nu\left(C_{G^{*}}(s)\right)=\alpha^{-}(a) \alpha^{+}(b) \cdot \beta(n-a-b),
$$

where $(a, b)$ are as above. Therefore, in case (i), the maximum of $\nu\left(C_{G^{*}}(s)\right)$ is attained for the values of $a, b$ as in the statement, and hence it is left to be shown that the maximum is not greater than this in cases (ii) and (iii).

Suppose that $n$ is odd. Then the maximum of $\alpha^{+}(a) \alpha^{-}(b) \beta(n-a-b)$ in case (ii) and of $\alpha^{+}(a) \alpha^{+}(b) \beta(n-a-b)$ in case (iii) is attained for $\left(a^{\prime}, 15\right)$ or $\left(15, b^{\prime}\right)$ with $a^{\prime}, b^{\prime}$ even; in addition, $\alpha^{-}(15)=\alpha^{+}(15)$. By swapping $V_{1}, V_{2}$ if necessary, we arrive at the case with $a=15$ and $V_{1}$ of Witt defect 1 , that is, at case (i). So the result follows for $n$ odd.

Let $n$ be even. We show that case (ii) can be ignored. Indeed, the maximum of the functions $\alpha^{+}(a) \alpha^{-}(b) \beta(n-a-b)$ and $\alpha^{-}(a) \alpha^{+}(b) \beta(n-a-b)$ is attained for $(a, b)=(16,16)$ if $n \equiv 0 \bmod 4$, and for $(a, b)=(16,14)$ and $(14,16)$ if $n \equiv 2 \bmod 4$. So the two maxima coincide.

It remains to compare the maxima of $\left|\mathcal{E}_{S}\right|$ in (i) and (iii) for $n$ even. In case (i), this is $\alpha^{-}(16) \alpha^{+}(16) \beta(n-32)$ if $n \equiv 0 \bmod 4$, and $\alpha^{-}(16) \alpha^{+}(14) \cdot \beta(n-30)$ if $n \equiv 2 \bmod 4$. In case (iii),

$$
v\left(C_{G^{*}}(s)\right)=\alpha^{+}(a) \alpha^{+}(b) v\left(C_{\mathrm{SO}(W)}\left(s^{\prime}\right)\right) \leq \alpha^{+}(a) \alpha^{+}(b) \beta(n-a-b) .
$$

Suppose first that $n-a-b$ is odd. Then (see Theorem 1.2)

$$
\begin{array}{cc}
\beta(n-a-b)=7 \beta(n-5-a-b) & \text { if } n-a-b \equiv 1 \bmod 4, \\
\beta^{\prime}(n-a-b)=77 \beta(n-11-a-b) & \text { if } n-a-b \equiv 3 \bmod 4 .
\end{array}
$$


In the latter case, Proposition 5.8 applied to $\alpha^{+}(a) \alpha^{+}(b) \beta(n-11-a-b)$ if $n-11 \geq 32$ yields that $\alpha^{+}(a) \alpha^{+}(b) \beta(n-11-a-b)$ does not exceed the following values:

$$
\begin{array}{ll}
77 \alpha^{+}(15) \alpha^{+}(14) \beta(n-40)=385945560 \cdot 5^{(n-40) / 4} & \text { if } n \equiv 0 \bmod 4, \\
77 \alpha^{+}(15) \alpha^{+}(16) \beta(n-42)=863963100 \cdot 5^{(n-42) / 4} & \text { if } n \equiv 2 \bmod 4 .
\end{array}
$$

A similar statement can be written for $\alpha^{+}(a) \alpha^{+}(b) \beta(n-5-a-b)$, but one observes from Theorem 1.2 that $\beta(n-5-a-b)=\beta(6) \beta(n-11-a-b)$ provided $n-11-a-b \geq 0$ and $n-11-a-b \equiv 0 \bmod 4$. As $\beta(6)=11$, we obtain the same values as above for the maximum of $\alpha^{+}(a) \alpha^{+}(b) \beta(n-5-a-b)$.

Note that

$$
\beta(n-i)=5^{(n-i) / 4} \quad \text { for } i=40,42
$$

above as $n-i \equiv 0 \bmod 4$ in each case. (Observe that we do not apply Proposition 5.8 directly to the function $\alpha^{+}(a) \alpha^{+}(b) \beta(n-a-b)$ as $a+b$ is here odd, whereas the maximum of this function with $n$ even is attained with $a+b$ even.)

Let $n-a-b$ be even. Then we have $v\left(C_{\mathrm{SO}(W)}\left(s^{\prime}\right)\right) \leq \beta^{\prime}(n-a-b)$. Recall (Lemma 2.3) that

$$
\beta^{\prime}(n-a-b)=\left\{\begin{aligned}
539 \cdot \beta(n-a-b-16) & \text { if } n-a-b \\
n-a-b & \geq 0 \bmod 4, \\
49 \cdot \beta(n-a-b-10)) & \text { if } n-a-b=2 \bmod 4, \\
n-a-b & >6 .
\end{aligned}\right.
$$

So

$$
\alpha^{+}(a) \alpha^{+}(b) \beta^{\prime}(n-a-b)=\left\{\begin{array}{c}
539 \cdot \alpha^{+}(a) \alpha^{+}(b) \beta(n-a-b-16), \\
49 \cdot \alpha^{+}(a) \alpha^{+}(b) \beta(n-a-b-10)
\end{array}\right.
$$

accordingly; by Proposition 5.6, if $n-16 \geq 18$, respectively, $n-10 \geq 18$, then the maximum of these functions is attained for $(a, b)=(16,16)$ if $n \equiv n-16 \equiv 0$ $\bmod 4$, respectively, for $(a, b)=(16,14)$ if $n-10 \equiv 0 \bmod 4$ (i.e., $n \equiv 2 \bmod 4)$. In fact, it suffices to record an upper bound for the case where $n-a \equiv 0 \bmod 4$ as $\beta(n-a-b-10)=\beta(6) \beta(n-a-b-16)=11 \beta(n-a-b-16)$ provided $n-a-b-16 \geq 0$ and $n-a-b-16 \equiv 0 \bmod 4$.

Thus, if $n-a-b$ is even, then $\alpha^{+}(a) \alpha^{+}(b) \beta^{\prime}(n-a-b)$ does not exceed the following values:

$$
\begin{array}{ll}
539 \alpha^{+}(16) \alpha^{+}(16) \beta(n-48)=9104841900 \cdot 5^{(n-48) / 4} & \text { if } n \equiv 0 \bmod 4, \\
539 \alpha^{+}(16) \alpha^{+}(14) \beta(n-46)=4067272440 \cdot 5^{(n-46) / 4} & \text { if } n \equiv 2 \bmod 4 .
\end{array}
$$


These must be compared with

$$
\begin{array}{ll}
\alpha^{-}(16) \alpha^{+}(16) \beta(n-32)=16711260 \cdot 5^{(n-32) / 4} & \text { if } n \equiv 0 \bmod 4, \\
\alpha^{-}(16) \alpha^{+}(14) \beta(n-30)=7465176 \cdot 5^{(n-30) / 4} & \text { if } n \equiv 2 \bmod 4 .
\end{array}
$$

For $n \equiv 0 \bmod 4$, we have

$$
\begin{aligned}
& \alpha^{-}(16) \alpha^{+}(16) \beta(n-32)=417781500 \beta(n-40) \\
& \quad>77 \alpha^{+}(15) \alpha^{+}(14) \beta(n-40)=385945560 \beta(n-40) \\
& \quad>539 \alpha^{+}(16) \alpha^{+}(16) \beta(n-48)=364193676 \beta(n-40) .
\end{aligned}
$$

For $n \equiv 2 \bmod 4$, we have

$$
\begin{aligned}
& \alpha^{-}(16) \alpha^{+}(14) \beta(n-30)=933147000 \beta(n-42) \\
& \quad>77 \alpha^{+}(15) \alpha^{+}(16) \beta(n-42)=863963100 \beta(n-42) \\
& \quad>539 \alpha^{+}(16) \alpha^{+}(14) \beta(n-46)=813454488 \beta(n-42) .
\end{aligned}
$$

This completes the proof of the main statement.

Finally, by Lemma 4.10, the bound in Theorem 1.5 is attained, proving the additional assertion.

Proof of Theorems 1.5 and 1.1. Theorem 1.5 follows from Theorems 7.2, 7.3, 7.4 and 7.5 for $q$ odd. For $q$ even, use Theorems 6.2, 6.3; note that the result for the group $G^{*}=\operatorname{Spin}_{2 n+1}(q), q$ even (not considered in Theorems 6.3), are identical to those for $G^{*}=\operatorname{Sp}_{2 n}(q)$ due to the comments after Lemma 2.7. Theorem 1.1 follows from Theorem 1.5 by elementary straightforward computations.

\section{A Appendix: The numerical data}

\begin{tabular}{rrrrr}
\hline$n$ & $\beta(n)$ & $\alpha(n)$ & $\alpha^{+}(n)$ & $\alpha^{-}(n)$ \\
\hline 1 & 1 & 2 & 1 & 1 \\
2 & 2 & 6 & 4 & 2 \\
3 & 3 & 12 & 5 & 5 \\
4 & 5 & 25 & 14 & 10 \\
5 & 7 & 46 & 20 & 20 \\
6 & 11 & 86 & 42 & 36 \\
\hline
\end{tabular}

Table 2. $\alpha(n), \alpha^{+}(n)$ and $\alpha^{-}(n)$ for $1 \leq n \leq 43$ 


\begin{tabular}{|c|c|c|c|c|}
\hline$n$ & $\beta(n)$ & $\alpha(n)$ & $\alpha^{+}(n)$ & $\alpha^{-}(n)$ \\
\hline 7 & 15 & 148 & 65 & 65 \\
\hline 8 & 25 & 255 & 120 & 110 \\
\hline 9 & 35 & 420 & 186 & 186 \\
\hline 10 & 55 & 686 & 316 & 302 \\
\hline 11 & 77 & 1088 & 486 & 486 \\
\hline 12 & 125 & 1712 & 784 & 762 \\
\hline 13 & 175 & 2634 & 1185 & 1185 \\
\hline 14 & 275 & 4020 & 1836 & 1806 \\
\hline 15 & 385 & 6036 & 2730 & 2730 \\
\hline 16 & 625 & 8988 & 4110 & 4066 \\
\hline 17 & 875 & 13214 & 6007 & 6007 \\
\hline 18 & 1375 & 19282 & 8830 & 8770 \\
\hline 19 & 1925 & 27840 & 12711 & 12711 \\
\hline 20 & 3125 & 39923 & 18326 & 18242 \\
\hline 21 & 4375 & 56750 & 26007 & 26007 \\
\hline 22 & 6875 & 86160 & 36884 & 36772 \\
\hline 23 & 9625 & 112384 & 51675 & 51675 \\
\hline 24 & 15625 & 156660 & 72260 & 72106 \\
\hline 25 & 21875 & 216958 & 100058 & 100058 \\
\hline 26 & 34375 & 298894 & 138186 & 137984 \\
\hline 27 & 48125 & 409420 & 189322 & 189322 \\
\hline 28 & 78125 & 558119 & 258610 & 258340 \\
\hline 29 & 109375 & 756950 & 350877 & 350877 \\
\hline 30 & 171875 & 1022090 & 474580 & 474228 \\
\hline 31 & 240625 & 1373760 & 638203 & 638203 \\
\hline 32 & 390625 & 1838932 & 855536 & 855074 \\
\hline 33 & 546875 & 2451366 & 1141125 & 1141125 \\
\hline 34 & 859375 & 3255480 & 1517336 & 1516742 \\
\hline 35 & 1203125 & 4306920 & 2008633 & 2008633 \\
\hline 36 & 1953125 & 5678104 & 2651020 & 2650250 \\
\hline 37 & 2734375 & 7459634 & 3484969 & 3484969 \\
\hline 38 & 4296875 & 9768386 & 4568010 & 4567030 \\
\hline 39 & 6015625 & 12750360 & 5966183 & 5966183 \\
\hline 40 & 9765625 & 16592332 & 7770754 & 7769500 \\
\hline 41 & 13671875 & 21527228 & 10088066 & 10088066 \\
\hline 42 & 21484375 & 27850932 & 13061880 & 13060296 \\
\hline 43 & 30078125 & 35931532 & 16861595 & 16861595 \\
\hline
\end{tabular}

Table 2. continued 


\begin{tabular}{|c|c|c|}
\hline$n$ & $\alpha(a) \alpha(b) \beta(n-a-b)$ & $\alpha(a) \alpha^{+}(b) \beta(n-a-b)$ \\
\hline 1 & $\alpha(1) \alpha(0)=2$ & $\alpha(1) \alpha^{+}(0)=2$ \\
\hline 2 & $\alpha(2) \alpha(0)=6$ & $\alpha(2) \alpha^{+}(0)=6$ \\
\hline 3 & $\alpha(2) \alpha(1)=\alpha(3) \alpha(0)=12$ & $\alpha(3) \alpha^{+}(0)=12$ \\
\hline 4 & $\alpha(2) \alpha(2)=36$ & $\alpha(4) \alpha^{+}(0)=25$ \\
\hline 5 & $\alpha(3) \alpha(2)=72$ & $\alpha(3) \alpha^{+}(2)=48$ \\
\hline 6 & $\alpha(4) \alpha(2)=150$ & $\alpha(4) \alpha^{+}(2)=100$ \\
\hline 7 & $\alpha(4) \alpha(3)=300$ & $\alpha(5) \alpha^{+}(2)=184$ \\
\hline 8 & $\alpha(4) \alpha(4)=625$ & $\alpha(4) \alpha^{+}(4)=350$ \\
\hline 9 & $\alpha(5) \alpha(4)=1150$ & $\alpha(5) \alpha^{+}(4)=644$ \\
\hline 10 & $\alpha(6) \alpha(4)=2150$ & $\alpha(6) \alpha^{+}(4)=1204$ \\
\hline 11 & $\alpha(6) \alpha(5)=3956$ & $\alpha(7) \alpha^{+}(4)=2072$ \\
\hline 12 & $\alpha(6) \alpha(6)=7396$ & $\alpha(6) \alpha^{+}(6)=3612$ \\
\hline 13 & $\alpha(7) \alpha(6)=12728$ & $\alpha(7) \alpha^{+}(6)=6216$ \\
\hline 14 & $\alpha(8) \alpha(6)=21930$ & $\alpha(8) \alpha^{+}(6)=10710$ \\
\hline 15 & $\alpha(8) \alpha(7)=37740$ & $\alpha(7) \alpha^{+}(8)=17760$ \\
\hline 16 & $\alpha(8) \alpha(8)=65025$ & $\alpha(8) \alpha^{+}(8)=30600$ \\
\hline 17 & $\alpha(9) \alpha(8)=107100$ & $\alpha(9) \alpha^{+}(8)=50400$ \\
\hline 18 & $\alpha(9) \alpha(9)=176400$ & $\alpha(10) \alpha^{+}(8)=82320$ \\
\hline 19 & $\alpha(10) \alpha(9)=288120$ & $\alpha(9) \alpha^{+}(10)=132720$ \\
\hline 20 & $\alpha(10) \alpha(10)=470596$ & $\alpha(10) \alpha^{+}(10)=216776$ \\
\hline 21 & $\alpha(11) \alpha(10)=746368$ & $\alpha(11) \alpha^{+}(10)=343808$ \\
\hline 22 & $\alpha(11) \alpha(11)=1183744$ & $\alpha(12) \alpha^{+}(10)=540992$ \\
\hline 23 & $\alpha(12) \alpha(11)=1862656$ & $\alpha(11) \alpha^{+}(12)=852992$ \\
\hline 24 & $\alpha(12) \alpha(12)=2930944$ & $\alpha(12) \alpha^{+}(12)=1342208$ \\
\hline 25 & $\alpha(13) \alpha(12)=4509408$ & $\alpha(13) \alpha^{+}(12)=2065056$ \\
\hline 26 & $\alpha(13) \alpha(13)=6937956$ & $\alpha(14) \alpha^{+}(12)=3151680$ \\
\hline 27 & $\alpha(14) \alpha(13)=10588680$ & $\alpha(13) \alpha^{+}(14)=4836024$ \\
\hline 28 & $\alpha(14) \alpha(14)=16160400$ & $\alpha(14) \alpha^{+}(14)=7380720$ \\
\hline 29 & $\alpha(15) \alpha(14)=24264720$ & $\alpha(15) \alpha^{+}(14)=11082096$ \\
\hline 30 & $\alpha(15) \alpha(15)=36433296$ & $\alpha(14) \alpha^{+}(16)=16522200$ \\
\hline 31 & $\alpha(16) \alpha(15)=54251568$ & $\alpha(15) \alpha^{+}(16)=24807960$ \\
\hline 32 & $\alpha(14) \alpha(14) \beta(4)=80802000$ & $\alpha(16) \alpha^{+}(16)=36940680$ \\
\hline 33 & $\alpha(15) \alpha(14) \beta(4)=121323600$ & $\alpha(15) \alpha^{+}(14) \beta(4)=55410480$ \\
\hline
\end{tabular}

Table 3. Maxima of $\alpha(a) \alpha(b) \beta(n-a-b)$ and $\alpha(a) \alpha^{+}(b) \beta(n-a-b)$ for $1 \leq n \leq 33$ 


\begin{tabular}{|c|c|c|}
\hline$n$ & $\alpha^{+}(a) \alpha^{+}(b) \beta(n-a-b)$ & $\alpha^{-}(a) \alpha^{+}(b) \beta(n-a-b)$ \\
\hline 1 & $\alpha^{+}(1) \alpha^{+}(0)=1$ & $\alpha^{-}(1) \alpha^{+}(0)=1$ \\
\hline 2 & $\alpha^{+}(2) \alpha^{+}(0)=4$ & $\alpha^{-}(0) \alpha^{+}(2)=4$ \\
\hline 3 & $\alpha^{+}(3) \alpha^{+}(0)=5$ & $\alpha^{-}(3) \alpha^{+}(0)=5$ \\
\hline 4 & $\alpha^{+}(2) \alpha^{+}(2)=16$ & $\alpha^{-}(0) \alpha^{+}(4)=14$ \\
\hline 5 & $\alpha^{+}(3) \alpha^{+}(2)=\alpha^{+}(5) \alpha^{+}(0)=20$ & $\alpha^{-}(3) \alpha^{+}(2)=\alpha^{-}(5) \alpha^{+}(0)=20$ \\
\hline 6 & $\alpha^{+}(4) \alpha^{+}(2)=56$ & $\alpha^{-}(0) \alpha^{+}(6)=42$ \\
\hline 7 & $\alpha^{+}(5) \alpha^{+}(2)=80$ & $\alpha^{-}(5) \alpha^{+}(2)=80$ \\
\hline 8 & $\alpha^{+}(4) \alpha^{+}(4)=196$ & $\alpha^{-}(6) \alpha^{+}(2)=144$ \\
\hline 9 & $\alpha^{+}(5) \alpha^{+}(4)=280$ & $\alpha^{-}(5) \alpha^{+}(4)=280$ \\
\hline 10 & $\alpha^{+}(6) \alpha^{+}(4)=588$ & $\alpha^{-}(6) \alpha^{+}(4)=504$ \\
\hline 11 & $\alpha^{+}(7) \alpha^{+}(4)=910$ & $\alpha^{-}(7) \alpha^{+}(4)=910$ \\
\hline 12 & $\alpha^{+}(6) \alpha^{+}(6)=1764$ & $\alpha^{-}(8) \alpha^{+}(4)=1540$ \\
\hline 13 & $\alpha^{+}(7) \alpha^{+}(6)=2730$ & $\alpha^{-}(7) \alpha^{+}(6)=2730$ \\
\hline 14 & $\alpha^{+}(8) \alpha^{+}(6)=5040$ & $\alpha^{-}(8) \alpha^{+}(6)=4620$ \\
\hline 15 & $\alpha^{+}(9) \alpha^{+}(6)=7812$ & $\alpha^{-}(9) \alpha^{+}(6)=7812$ \\
\hline 16 & $\alpha^{+}(8) \alpha^{+}(8)=14400$ & $\alpha^{-}(8) \alpha^{+}(8)=13200$ \\
\hline 17 & $\alpha^{+}(9) \alpha^{+}(8)=22320$ & $\alpha^{-}(9) \alpha^{+}(8)=22320$ \\
\hline 18 & $\alpha^{+}(10) \alpha^{+}(8)=37920$ & $\alpha^{-}(10) \alpha^{+}(8)=36240$ \\
\hline 19 & $\alpha^{+}(9) \alpha^{+}(10)=58776$ & $\alpha^{-}(9) \alpha^{+}(10)=58776$ \\
\hline 20 & $\alpha^{+}(10) \alpha^{+}(10)=99856$ & $\alpha^{-}(10) \alpha^{+}(10)=95432$ \\
\hline 21 & $\alpha^{+}(11) \alpha^{+}(10)=153576$ & $\alpha^{-}(11) \alpha^{+}(10)=153576$ \\
\hline 22 & $\alpha^{+}(12) \alpha^{+}(10)=247744$ & $\alpha^{-}(12) \alpha^{+}(10)=240792$ \\
\hline 23 & $\alpha^{+}(11) \alpha^{+}(12)=381024$ & $\alpha^{-}(11) \alpha^{+}(12)=381024$ \\
\hline 24 & $\alpha^{+}(12) \alpha^{+}(12)=614656$ & $\alpha^{-}(12) \alpha^{+}(12)=397408$ \\
\hline 25 & $\alpha^{+}(13) \alpha^{+}(12)=929040$ & $\alpha^{-}(13) \alpha^{+}(12)=929040$ \\
\hline 26 & $\alpha^{+}(14) \alpha^{+}(12)=1439424$ & $\alpha^{-}(14) \alpha^{+}(12)=1415904$ \\
\hline 27 & $\alpha^{+}(13) \alpha^{+}(14)=2175660$ & $\alpha^{-}(13) \alpha^{+}(14)=2175660$ \\
\hline 28 & $\alpha^{+}(14) \alpha^{+}(14)=3370896$ & $\alpha^{-}(14) \alpha^{+}(14)=3315816$ \\
\hline 29 & $\alpha^{+}(15) \alpha^{+}(14)=5012280$ & $\alpha^{-}(15) \alpha^{+}(14)=5012280$ \\
\hline 30 & $\alpha^{+}(16) \alpha^{+}(14)=7545960$ & $\alpha^{-}(16) \alpha^{+}(14)=7465176$ \\
\hline 31 & $\alpha^{+}(15) \alpha^{+}(16)=11220300$ & $\alpha^{-}(15) \alpha^{+}(16)=11220300$ \\
\hline 32 & $\alpha^{+}(16) \alpha^{+}(16)=16892100$ & $\alpha^{-}(16) \alpha^{+}(16)=16711260$ \\
\hline 33 & $\alpha^{+}(15) \alpha^{+}(14) \beta(4)=25061400$ & $\alpha^{-}(15) \alpha^{+}(14) \beta(4)=25061400$ \\
\hline
\end{tabular}

Table 4. Maxima of $\alpha^{+}(a) \alpha^{+}(b) \beta(n-a-b)$ and $\alpha^{-}(a) \alpha^{+}(b) \beta(n-a-b)$ for $1 \leq n \leq 33$ 
Remark. For $n \leq 31$, Table 3 also implies the maxima of $\alpha(a) \alpha(b)$ for $a, b$ such that $a+b=n$. For $n=32$, the maximum is attained at $(a, b)=(16,16)$ with value 80784144 ; for $n=33$, the maximum is attained at $(a, b)=(16,17)$ with value 118767432 .

Acknowledgments. We are very grateful to Gunter Malle for his comments on the original manuscript which were helpful in correcting inaccuracies and improving upon the presentation. Thanks also go to the referee for some useful comments on the last version.

\section{Bibliography}

[1] C. Bessenrodt and K. Ono, Maximal multiplicative properties of partitions, Ann. Comb. 20 (2016), no. 1, 59-64.

[2] M. Cabanes and M. Enguehard, Representation Theory of Finite Reductive Groups, New Math. Monogr. 1, Cambridge University, Cambridge, 2004.

[3] R. W. Carter, Finite Groups of Lie Type, Pure Appl. Mathematics (New York), John Wiley \& Sons, New York, 1985.

[4] J. A. Dieudonné, La géométrie des groupes classiques, 3rd ed., Springer, Berlin, 1971.

[5] F. Digne and J. Michel, Representations of Finite Groups of Lie Type, London Math. Soc. Stud. Texts 21, Cambridge University, Cambridge, 1991.

[6] P. Fong and B. Srinivasan, The blocks of finite classical groups, J. Reine Angew. Math. 396 (1989), 122-191.

[7] B. Huppert, Singer-Zyklen in klassischen Gruppen, Math. Z. 117 (1970), 141-150.

[8] P. Kleidman and M. Liebeck, The Subgroup Structure of the Finite Classical Groups, London Math. Soc. Lecture Note Ser. 129, Cambridge University, Cambridge, 1990.

[9] E. Kowalski, The Large Sieve and its Applications, Cambridge Tracts in Math. 175, Cambridge University, Cambridge, 2008.

[10] M. W. Liebeck and A. Shalev, Character degrees and random walks in finite groups of Lie type, Proc. Lond. Math. Soc. (3) 90 (2005), no. 1, 61-86.

[11] G. Lusztig, Irreducible representations of finite classical groups, Invent. Math. 43 (1977), no. 2, 125-175.

[12] G. Malle and D. Testerman, Linear Algebraic Groups and Finite Groups of Lie Type, Cambridge Stud. Adv. Math. 133, Cambridge University, Cambridge, 2011.

[13] J. Siemons and A. Zalesski, Regular orbits of cyclic subgroups in permutation representations of certain simple groups, J. Algebra 256 (2002), no. 2, 611-625. 
[14] P. Spiga and A. Zalesski, A uniform upper bound for the character degree sums and Gelfand-Graev-like characters for finite simple groups, in: Group Theory, Combinatorics, and Computing, Contemp. Math. 611, American Mathematical Society, Providence (2014), 169-187.

[15] T. A. Springer and R. Steinberg, Conjugacy classes, in: Seminar on Algebraic Groups and Related Finite Groups, Lecture Notes in Math. 131, Springer, Berlin (1970), 167-266.

[16] A. E. Zalesski, On the Steinberg character of an orthogonal group over a finite field, J. Algebra Appl. 13 (2014), no. 7, Article ID 1450033.

Received April 6, 2020; revised September 6, 2020.

\section{Author information}

Corresponding author:

Christine Bessenrodt, Institute for Algebra, Number Theory and Discrete Mathematics, Faculty of Mathematics and Physics, Leibniz University Hannover, Welfengarten 1, 30167, Hannover, Germany.

E-mail: bessen@math.uni-hannover.de

Alexandre Zalesski, Department of Physics, Mathematics and Informatics, National Academy of Sciences of Belarus, 66 Nezalejnasti prospekt, 220072, Minsk, Belarus.

E-mail: alexandre.zalesski@gmail.com 